\title{
Approximation of a general singular vertex coupling in quantum graphs
}

\author{
Taksu Cheon ${ }^{\mathrm{a}}$, Pavel Exner ${ }^{\mathrm{b}, \mathrm{c}}$, Ondřej Turek ${ }^{\mathrm{b}, \mathrm{d}}$ \\ ${ }^{a}$ Laboratory of Physics, Kochi University of Technology \\ Tosa Yamada, Kochi 782-8502, Japan \\ ${ }^{b}$ Doppler Institute for Mathematical Physics and Applied Mathematics, Czech Technical University \\ Břehová 7, 11519 Prague, Czech Republic \\ ${ }^{c}$ Department of Theoretical Physics, Nuclear Physics Institute, Czech Academy of Sciences \\ 25068 Řež near Prague, Czech Republic \\ ${ }^{d}$ Department of Mathematics, Faculty of Nuclear Sciences and Physical Engineering, Czech Technical University \\ Trojanova 13, 12000 Prague, Czech Republic
}

\begin{abstract}
The longstanding open problem of approximating all singular vertex couplings in a quantum graph is solved. We present a construction in which the edges are decoupled; an each pair of their endpoints is joined by an edge carrying a $\delta$ potential and a vector potential coupled to the "loose" edges by a $\delta$ coupling. It is shown that if the lengths of the connecting edges shrink to zero and the potentials are properly scaled, the limit can yield any prescribed singular vertex coupling, and moreover, that such an approximation converges in the norm-resolvent sense.
\end{abstract}

Key words: quantum graphs, boundary conditions, singular vertex coupling, quantum wires PACS: 03.65.-w, 03.65.Db, 73.21.Hb

\section{Introduction}

While the origin of the idea to investigate quantum mechanics of particles confined to a graph was conceived originally to address to a particular physical problem, namely the spectra of aromatic hydrocarbons [1], the motivation was quickly lost and for a long time the problem remained rather an obscure textbook example. This changed in the last two decades when the progress of microfabrication techniques made graph-shaped structures of submicron sizes technologically important. This generated an intense interest to investigation of quantum graph models which went beyond the needs of practical applications, since these models proved to be an excellent laboratory to study various properties of quantum systems. The literature on quantum graphs is nowadays huge; we limit ourselves to mentioning the recent volume [2] where many concepts are discussed and a rich bibliography can be found.

The essential component of quantum graph models is the wavefunction coupling in the vertices. While often the most simple matching conditions (dubbed free, Kirchhoff, or Neumann) or the slightly more general $\delta$ coupling in which the functions are continuous in the vertex are

Email addresses: taksu. cheon@kochi-tech.ac.jp (Taksu Cheon), exner@ujf.cas.cz (Pavel Exner), turekond@fjfi.cvut.cz (Ondřej Turek)

Preprint submitted to Elsevier 
used, these cases represent just a tiny subset of all admissible couplings. The family of the latter is determined by the requirement that the corresponding Hamiltonian is a self-adjoint operator, or in physical language, that the probability current is conserved at the vertices. It is not difficult to find all the admissible conditions mathematically; if the vertex joins $n$ edges they contain $n^{2}$ free parameters, and with exception of the one-parameter subfamily mentioned above they are all singular in the sense that the wavefunctions are discontinuous at the vertex.

What is much less clear is the physical meaning of such conditions. It is longstanding open problem whether and in what sense one can approximate all the singular couplings by regular ones depending on suitable parameters, and the aim of the present paper is to answer this question by presenting such a construction, minimal in a natural sense using $n^{2}$ real parameters, and to show that the closeness is achieved in the norm-resolvent sense, so the convergence of all types of the spectra and the corresponding eigenprojections is guaranteed.

The key idea comes from a paper of one of us with Shigehara [3] which showed that a combination of regular point interactions on a line approaching each other with the coupling scaled in a particular way w.r.t. the interaction distance can produce a singular point interaction. Later it was demonstrated [4] that the convergence in this model is norm-resolvent and the scaling choice is highly non-generic. The idea was applied by two of us to the simplest singular coupling, the so-called $\delta_{\mathrm{s}}^{\prime}$, in [5] and was demonstrated to work; the question was how much it can be extended. Two other of us examined it [6] and found that with a larger number of regular interactions one can deal with families described by $2 n$ parameters, and changing locally the approximating graph topology one can deal with all the couplings invariant with respect to the time reversal which form an $\left(\begin{array}{c}n+1 \\ 2\end{array}\right)$-parameter subset.

It was clear that to proceed beyond the time-reversal symmetry one has to involve vector potentials similarly as it is was done in the simplest situation in [7]. In this paper we present such a construction which contains parameters breaking the symmetry and which at the same time is more elegant than that of [6] in the sense that the needed "ornamentation" of the graph is minimal: we disconnect the $n$ edges at the vertex and join each pair of the so obtained free ends by an additional edge which shrinks to a point in the limit. The number of parameters leans on the decomposition $n^{2}=n+2\left(\begin{array}{l}n \\ 2\end{array}\right)$, where the first summand, $n$, corresponds to $\delta$ couplings of the "outer" edge endpoints with those of the added shrinking ones. The second summand can be considered as $\left(\begin{array}{l}n \\ 2\end{array}\right)$ times two parameters: one is a $\delta$ potential placed at the edge, the other is a vector potential supported by it.

Our result shows that any singular vertex coupling can be approximated by a graph in which the vertex is replaced by a local graph structure in combination with local regular interactions and local magnetic fields. This opens way to constructing "structured" vertices tailored to the desired conductivity properties, even tunable ones, if the interactions are controlled by gate electrodes, however, we are not going to elaborate such approximations further in this paper.

We have to note for completeness that the problem of understanding vertex couplings has also other aspects. The approximating object needs not to be a graph but can be another geometrical structure. A lot of attention was paid to the situation of "fat graphs", or networks of this tubes built around the graph skeleton. The two approaches can be combined, for instance, by "lifting" the graph results to fat graphs. In this way approximations to $\delta$ and $\delta_{\mathrm{s}}^{\prime}$ couplings by suitable families of Schrödinger operators on such manifolds with Neumann boundaries were recently demonstrated in [8]. The results of this paper can be similarly "lifted" to manifolds; that will be the subject of a subsequent work.

Let us review briefly the contents of the paper. In the next section we gather the needed 
preliminary information. We review the information about vertex couplings and derive a new parametrization of a general coupling suitable for our purposes. In Section 3 we describe in detail the approximation sketched briefly above and show that on a heuristic level it converges to a chosen vertex coupling. Finally, in the last section we present and prove our main result showing that the said convergence is not only formal but it is valid also in the norm-resolvent sense.

\section{Vertex coupling in quantum graphs}

Let us first recall briefly a few basic notions; for a more detailed discussion we refer to the literature given in the introduction. The object of our interest are Schrödinger operators on metric graphs. A graph is conventionally identified with a family of vertices and edges; it is metric if each edge can be equipped with a distance, i.e. to be identified with a finite or semi-infinite interval.

We regard such a graph $\Gamma$ with edges $E_{1}, \ldots, E_{n}$ as a configuration space of a quantum mechanical system, i.e. we identify the orthogonal sum $\mathcal{H}=\bigoplus_{j=1}^{n} L^{2}\left(E_{j}\right)$ with the state Hilbert space and the wave function of a spinless particle "living" on $\Gamma$ can be written as the column $\Psi=\left(\psi_{1}, \psi_{2}, \ldots, \psi_{n}\right)^{T}$ with $\psi_{j} \in L^{2}\left(E_{j}\right)$. In the simplest case when no external fields are present the system Hamiltonian acts as $\left(H_{\Gamma} \Psi\right)_{j}=-\psi_{j}^{\prime \prime}$, with the domain consisting of functions from $W^{2,2}(\Gamma):=\bigoplus_{j=1}^{n} W^{2,2}\left(E_{j}\right)$. Not all such functions are admissible, though, in order to make the operator self-adjoint we have to require that appropriate boundary conditions are satisfied at the vertices of the graph.

We restrict our attention to the physically most interesting case when the boundary conditions are local, coupling values of the functions and derivatives is each vertex separately. Our aim is explain the meaning of a general vertex coupling using suitable approximations; the local character means that we can investigate how such a system behaves in the vicinity of a single vertex. A prototypical example of this situation is a star graph with one vertex in which a finite number of semi-infinite edges meet; this is the case we will mostly have in mind in the following.

Let us thus consider a graph vertex $V$ of degree $n$, i.e. with $n$ edges connected at $V$. We denote these edges by $E_{1}, \ldots, E_{n}$ and the components of the wave function values at them by $\psi_{1}\left(x_{1}\right), \ldots, \psi_{n}\left(x_{n}\right)$. We choose the coordinates at the edges in such a way that $x_{j} \geq 0$ for all $j=1, \ldots, n$, and the value $x_{j}=0$ corresponds to the vertex $V$. For notational simplicity we put $\Psi_{V}=\left(\psi_{1}(0), \ldots, \psi_{n}(0)\right)^{T}$ and $\Psi_{V}^{\prime}=\left(\psi_{1}^{\prime}(0), \ldots, \psi_{n}^{\prime}(0)\right)^{T}$. Since our Hamiltonian is a second-order differential operator, the sought boundary conditions will couple the above boundary values, their most general form being

$$
A \Psi_{V}+B \Psi_{V}^{\prime}=0
$$

where $A$ and $B$ are complex $n \times n$ matrices.

To ensure self-adjointness of the Hamiltonian, which is in physical terms equivalent to conservation of the probability current at the vertex $V$, the matrices $A$ and $B$ cannot be arbitrary but have to satisfy the following two conditions,

- $\operatorname{rank}(A \mid B)=n$,

- the matrix $A B^{*}$ is self-adjoint,

where $(A \mid B)$ denotes the $n \times 2 n$ matrix with $A, B$ forming the first and the second $n$ columns, respectively, as stated for the first time by Kostrykin and Schrader [9]. The relation (1) together 
with conditions (2) (for brevity, we will write (1)\&(2)) describe all possible vertex boundary conditions giving rise to a self-adjoint Hamiltonian; we will speak about admissible boundary conditions.

On the other hand, it is obvious that the formulation (1)\&(2) is non-unique in the sense that different pairs $\left(A_{1}, B_{1}\right),\left(A_{2}, B_{2}\right)$ may define the same vertex coupling, as $A, B$ can be equivalently replaced by $C A, C B$ for any regular matrix $C \in \mathbb{C}^{n, n}$. To overcome this ambiguity, Harmer [10], and independently Kostrykin and Schrader [11] proposed a unique form of the boundary conditions (1), namely

$$
(U-I) \Psi_{V}+\mathrm{i}(U+I) \Psi_{V}^{\prime}=0,
$$

where $U$ is a unitary $n \times n$ matrix. Note that in a more general context such conditions were known before [12], see also [13].

The natural parametrization (3) of the family of vertex couplings has several advantages in comparison to (1)\&(2), besides its uniqueness it also makes obvious how "large" the family is: since the unitary group $U(n)$ has $n^{2}$ real parameters, the same is true for vertex couplings in a quantum graph vertex of the degree $n$. Of course, this fact is also clear if one interprets the couplings from the viewpoint of self-adjoint extensions [14].

On the other hand, among the disadvantages of the formulation (3) one can mention its complexity: vertex couplings that are simple from the physical point of view may have a complicated description when expressed in terms of the condition (3). As an example, let us mention in the first place the $\delta$-coupling with a parameter $\alpha \in \mathbb{R}$, characterized by relations

$$
\psi_{j}(0)=\psi_{k}(0)=: \psi(0), \quad j, k=1 \ldots, n, \quad \sum_{j=1}^{n} \psi_{j}^{\prime}(0)=\alpha \psi(0),
$$

for which the matrix $U$ used in (3) has entries given by

$$
U_{j k}=\frac{2}{n+\mathrm{i} \alpha}-\delta_{j k},
$$

$\delta_{j k}$ being the Kronecker delta. When we substitute (5) into (3) and compare with (4) rewritten into a matrix form (1), we observe that the first formulation is not only more complicated with respect to the latter, but also contains complex values whereas the latter does not. This is a reason why it is often better to work with simpler expressions of the type (1)\&(2). Another aspect of this parametrization difference concerns the meaning of the parameters. Since the $n^{2}$ ones mentioned earlier are "encapsulated" in a unitary matrix, it is difficult to understand which role each of them plays.

On the other hand, both formulations (1)\&(2) and (3) have a common feature, namely that they have a form insensitive to a particular edge numbering. If the edges are permuted one has just to replace the matrices $A, B$ and $U$ by $\tilde{A}, \tilde{B}$ and $\tilde{U}$, respectively, obtained by the appropriate rearrangement of rows and columns. This may hide different ways in which the edges are coupled; it is easy to see that a particular attention should be paid to "singular" situations when the matrix $U$ has eigenvalue(s) equal to \pm 1 .

Since the type of the coupling will be important for the approximation we are going to construct, we will rewrite the vertex coupling conditions in another form which is again simple and unique but requires an appropriate edge numbering. This will be done in Theorem 2.1, before 
stating it we introduce several symbols that will be employed in the further text, namely

$$
\begin{aligned}
\mathbb{C}^{k, l} & - \text { the set of complex matrices with } k \text { rows and } l \text { columns, } \\
\hat{n} & - \text { the set }\{1,2, \ldots, n\}, \\
I^{(n)} & - \text { the identity matrix } n \times n .
\end{aligned}
$$

To be precise, let us remark that the term "numbering" with respect to the edges connected in the graph vertex of the degree $n$ means strictly numbering by the elements of the set $\hat{n}$.

Theorem 2.1. Let us consider a quantum graph vertex $V$ of the degree $n$.

(i) If $m \leq n, S \in \mathbb{C}^{m, m}$ is a self-adjoint matrix and $T \in \mathbb{C}^{m, n-m}$, then the equation

$$
\left(\begin{array}{cc}
I^{(m)} & T \\
0 & 0
\end{array}\right) \Psi_{V}^{\prime}=\left(\begin{array}{cc}
S & 0 \\
-T^{*} & I^{(n-m)}
\end{array}\right) \Psi_{V}
$$

expresses admissible boundary conditions. This statement holds true for any numbering of the edges.

(ii) For any vertex coupling there exist a number $m \leq n$ and a numbering of edges such that the coupling is described by the boundary conditions (6) with the uniquely given matrices $T \in \mathbb{C}^{m, n-m}$ and self-adjoint $S \in \mathbb{C}^{m, m}$.

(iii) Consider a quantum graph vertex of the degree $n$ with the numbering of the edges explicitly given; then there is a permutation $\Pi \in S_{n}$ such that the boundary conditions may be written in the modified form

$$
\left(\begin{array}{cc}
I^{(m)} & T \\
0 & 0
\end{array}\right) \tilde{\Psi}_{V}^{\prime}=\left(\begin{array}{cc}
S & 0 \\
-T^{*} & I^{(n-m)}
\end{array}\right) \tilde{\Psi}_{V}
$$

for

$$
\tilde{\Psi}_{V}=\left(\begin{array}{c}
\psi_{\Pi(1)}(0) \\
\vdots \\
\psi_{\Pi(n)}(0)
\end{array}\right), \quad \tilde{\Psi}_{V}^{\prime}=\left(\begin{array}{c}
\psi_{\Pi(1)}^{\prime}(0) \\
\vdots \\
\psi_{\Pi(n)}^{\prime}(0)
\end{array}\right),
$$

where the self-adjoint matrix $S \in \mathbb{C}^{m, m}$ and the matrix $T \in \mathbb{C}^{m, n-m}$ depend unambiguously on $\Pi$. This formulation of boundary conditions is in general not unique, since there may be different admissible permutations $\Pi$, but one can make it unique by choosing the lexicographically smallest permutation $\Pi$.

Proof. The claim (iii) is an immediate consequence of (ii) using a simultaneous permutation of elements in the vectors $\Psi_{V}$ and $\Psi_{V}^{\prime}$, so we have to prove the first two. As for (i), we have to show that the vertex coupling (1) with matrices

$$
A=\left(\begin{array}{cc}
-S & 0 \\
T^{*} & -I^{(n-m)}
\end{array}\right) \quad \text { and } \quad B=\left(\begin{array}{cc}
I^{(n)} & T \\
0 & 0
\end{array}\right),
$$

conform with (2). We have

$$
\operatorname{rank}\left(\begin{array}{cccc}
-S & 0 & I^{(m)} & T \\
T^{*} & -I^{(n-m)} & 0 & 0
\end{array}\right)=\underset{5}{\operatorname{rank}}\left(\begin{array}{cccc}
I^{(m)} & 0 & -S & T \\
0 & -I^{(n-m)} & T^{*} & 0
\end{array}\right)=n
$$


and

$$
\left(\begin{array}{cc}
-S & 0 \\
T^{*} & -I^{(n-m)}
\end{array}\right) \cdot\left(\begin{array}{cc}
I^{(n)} & T \\
0 & 0
\end{array}\right)^{*}=\left(\begin{array}{cc}
-S & 0 \\
0 & 0
\end{array}\right) ;
$$

the latter matrix is self-adjoint since $S=S^{*}$, thus (2) is satisfied.

Now we proceed to (ii). Consider a quantum graph vertex of the degree $n$ with an arbitrary fixed vertex coupling. Let $\Psi_{V}$ and $\Psi_{V}^{\prime}$ denote the vectors of values and derivatives of the wave function components at the edge ends; the order of the components is arbitrary but fixed and the same for both vectors. We know that the coupling can be described by boundary conditions (1) with some $A, B \in \mathbb{C}^{n, n}$ satisfying (2). Our aim is to find a number $m \leq n$, a certain numbering of the edges and matrices $S$ and $T$ such that the boundary conditions (1) are equivalent to (6). Moreover, we have to show that such a number $m$ is the only possible and that $S, T$ depend uniquely on the edge numbering.

When proceeding from (1) to (6), we may use exclusively manipulations that do not affect the meaning of the coupling, namely

- simultaneous permutation of columns of the matrices $A, B$ combined with corresponding simultaneous permutation of components in $\Psi_{V}$ and $\Psi_{V}^{\prime}$,

- multiplying the system from left by a regular matrix.

We see from (6) that $m$ is equal to the rank of the matrix applied at $\Psi_{V}^{\prime}$. We observe that the rank of this matrix, as well as of that applied at $\Psi_{V}$, is not influences by any of the manipulations mentioned above, hence it is obvious that $m=\operatorname{rank}(B)$ and that such a choice is the only possible, i.e. $m$ is unique.

Since $\operatorname{rank}(B)=m$ with $m \in\{0, \ldots, n\}$, there is an $m$-tuple of linearly independent columns of the matrix $B$; suppose that their indices are $j_{1}, \ldots, j_{m}$. We permute simultaneously the columns of $B$ and $A$ so that those with indices $j_{1}, \ldots, j_{m}$ are now at the positions $1, \ldots, m$, and the same we do with the components of the vectors $\Psi_{V}, \Psi_{V}^{\prime}$. Labelling the permuted matrices $A, B$ and vectors $\Psi_{V}, \Psi_{V}^{\prime}$ with tildes, we get

$$
\tilde{A} \tilde{\Psi}_{V}+\tilde{B} \tilde{\Psi}_{V}^{\prime}=0 .
$$

Since $\operatorname{rank}(\tilde{B})=\operatorname{rank}(B)=m$, there are $m$ rows of $\tilde{B}$ that are linearly independent, let their indices be $i_{1}, \ldots, i_{m}$, and $n-m$ rows that are linear combinations of the preceding ones. First we permute the rows in (8) so that those with indices $i_{1}, \ldots, i_{m}$ are put to the positions $1, \ldots, m$; note that it corresponds to a matrix multiplication of the whole system (8) by a permutation matrix (which is regular) from the left, i.e. an authorized manipulation. In this way we pass from $\tilde{A}$ and $\tilde{B}$ to matrices which we denote as $\check{A}$ and $\breve{B}$; it is obvious that this operation keeps the first $m$ columns of the matrix $\check{B}$ linearly independent.

In the next step we add to each of the last $n-m$ rows of $\breve{A} \tilde{\Psi}(0)+\breve{B} \tilde{\Psi}^{\prime}(0)=0$ such a linear combination of the first $m$ rows that all the last $n-m$ rows of $\breve{B}$ vanish. This is possible, because the last $n-m$ lines of $\breve{B}$ are linearly dependent on the first $m$ lines. It is easy to see that it is an authorized operation, not changing the meaning of the boundary conditions; the resulting matrices at the LHS will be denoted as $\hat{B}$ and $\hat{A}$, i.e.

$$
\hat{A} \tilde{\Psi}_{V}+\hat{B} \tilde{\Psi}_{V}^{\prime}=0 .
$$

From the construction described above we know that the matrix $\hat{B}$ has a block form,

$$
\hat{B}=\left(\begin{array}{cc}
\hat{\mathcal{B}}_{11} & \hat{\mathcal{B}}_{12} \\
0 & 0 \\
6 &
\end{array}\right),
$$


where $\hat{\mathcal{B}}_{11} \in \mathbb{C}^{m, m}$ and $\hat{\mathcal{B}}_{12} \in \mathbb{C}^{m, n-m}$; the square matrix $\hat{\mathcal{B}}_{11} \in \mathbb{C}^{m, m}$ is regular, because its columns are linearly independent. We proceed by multiplying the system (9) from the left by the matrix

$$
\left(\begin{array}{cc}
\hat{\mathcal{B}}_{11}^{-1} & 0 \\
0 & I^{(n-m)}
\end{array}\right)
$$

arriving at boundary conditions

$$
\left(\begin{array}{ll}
\mathcal{A}_{11} & \mathcal{A}_{12} \\
\mathcal{A}_{21} & \mathcal{A}_{22}
\end{array}\right) \tilde{\Psi}_{V}+\left(\begin{array}{cc}
I^{(m)} & \mathcal{B}_{12} \\
0 & 0
\end{array}\right) \tilde{\Psi}_{V}^{\prime}=0
$$

where $\mathcal{B}_{12}=\hat{\mathcal{B}}_{11}^{-1} \hat{\mathcal{B}}_{12}$.

Boundary conditions (10) are equivalent to (1), therefore they have to be admissible. In other words, the matrices $\left(\begin{array}{ll}\mathcal{A}_{11} & \mathcal{A}_{12} \\ \mathcal{A}_{21} & \mathcal{A}_{22}\end{array}\right)$ and $\left(\begin{array}{cc}I^{(m)} & \mathcal{B}_{12} \\ 0 & 0\end{array}\right)$ have to satisfy both the conditions (2), which we are now going to verify. Let us begin with the second one. We have

$$
\left(\begin{array}{ll}
\mathcal{A}_{11} & \mathcal{A}_{12} \\
\mathcal{A}_{21} & \mathcal{A}_{22}
\end{array}\right) \cdot\left(\begin{array}{ll}
I^{(m)} & 0 \\
\mathcal{B}_{12}^{*} & 0
\end{array}\right)=\left(\begin{array}{cc}
\mathcal{A}_{11}+\mathcal{A}_{12} \mathcal{B}_{12}^{*} & 0 \\
\mathcal{A}_{21}+\mathcal{A}_{22} \mathcal{B}_{12}^{*} & 0
\end{array}\right)
$$

and this matrix is self-adjoint if and only if $\mathcal{A}_{11}+\mathcal{A}_{12} \mathcal{B}_{12}^{*}$ is self adjoint and $\mathcal{A}_{21}+\mathcal{A}_{22} \mathcal{B}_{12}^{*}=0$. We infer that $\mathcal{A}_{21}=-\mathcal{A}_{22} \mathcal{B}_{12}^{*}$, hence condition (10) acquires the form

$$
\left(\begin{array}{cc}
\mathcal{A}_{11} & \mathcal{A}_{12} \\
-\mathcal{A}_{22} \mathcal{B}_{12}^{*} & \mathcal{A}_{22}
\end{array}\right) \tilde{\Psi}_{V}+\left(\begin{array}{cc}
I^{(m)} & \mathcal{B}_{12} \\
0 & 0
\end{array}\right) \tilde{\Psi}_{V}^{\prime}=0 .
$$

The first one of the conditions (2) says that

$$
\operatorname{rank}\left(\begin{array}{cccc}
\mathcal{A}_{11} & \mathcal{A}_{12} & I^{(m)} & \mathcal{B}_{12} \\
-\mathcal{A}_{22} \mathcal{B}_{12}^{*} & \mathcal{A}_{22} & 0 & 0
\end{array}\right)=n,
$$

hence $\operatorname{rank}\left(-\mathcal{A}_{22} \mathcal{B}_{12}^{*} \mid \mathcal{A}_{22}\right)=n-m$. Since $\left(-\mathcal{A}_{22} \mathcal{B}_{12}^{*} \mid \mathcal{A}_{22}\right)=-\mathcal{A}_{22} \cdot\left(\mathcal{B}_{12}^{*} \mid I^{(n-m)}\right)$ we obtain the condition $\operatorname{rank}\left(\mathcal{A}_{22}\right)=n-m$, i.e. $\mathcal{A}_{22}$ must be a regular matrix. It allows us to multiply the equation (11) from the left by the matrix

$$
\left(\begin{array}{cc}
I^{(m)} & -\mathcal{A}_{12} \mathcal{A}_{22}^{-1} \\
0 & -\mathcal{A}_{22}^{-1}
\end{array}\right)
$$

which is obviously well-defined and regular; this operation leads to the condition

$$
\left(\begin{array}{cc}
\mathcal{A}_{11}+\mathcal{A}_{12} \mathcal{B}_{12}^{*} & 0 \\
\mathcal{B}_{12}^{*} & -I^{(n-m)}
\end{array}\right) \tilde{\Psi}_{V}+\left(\begin{array}{cc}
I^{(m)} & \mathcal{B}_{12} \\
0 & 0
\end{array}\right) \tilde{\Psi}_{V}^{\prime}=0 .
$$

If follows from our previous considerations that the square matrix $\mathcal{A}_{11}+\mathcal{A}_{12} \mathcal{B}_{12}^{*}$ is self-adjoint. If we denote it as $-S$, rename the block $\mathcal{B}_{12}$ as $T$ and transfer the term containing $\tilde{\Psi}_{V}^{\prime}$ to the right hand side, we arrive at boundary conditions

$$
\left(\begin{array}{cc}
I^{(m)} & T \\
0 & 0
\end{array}\right) \tilde{\Psi}_{V}^{\prime}=\left(\begin{array}{cc}
S & 0 \\
-T^{*} & I^{(n-m)}
\end{array}\right) \tilde{\Psi}_{V} .
$$


The order of components in $\tilde{\Psi}_{V}$ and $\tilde{\Psi}_{V}^{\prime}$ determines just the appropriate numbering, in other words, the vectors $\tilde{\Psi}_{V}$ and $\tilde{\Psi}_{V}^{\prime}$ represent exactly what we understood by $\Psi_{V}$ and $\Psi_{V}^{\prime}$ in the formulation of the theorem.

Finally, the uniqueness of the matrices $S$ and $T$ with respect to the choice of the permutation $\Pi$ is a consequence of the presence of the blocks $I^{(m)}$ and $I^{(n-m)}$. First of all, the block $I^{(n-m)}$ implies that there is only one possible $T$, otherwise the conditions for $\tilde{\psi}_{m+1}^{\prime}, \ldots, \tilde{\psi}_{n}^{\prime}$ would change, and next, the block $I^{(m)}$ together with the uniqueness of $T$ implies that there is only one possible $S$, otherwise the conditions for $\tilde{\psi}_{1}, \ldots, \tilde{\psi}_{m}$ would change.

Remark 2.2. The expression (7) implies, in particular, that if $B$ has not full rank, the number of real numbers parametrizing the vertex coupling (1) is reduced from $n^{2}$ to at most $m(2 n-m)=$ $n^{2}-(n-m)^{2}$, where $m=\operatorname{rank}(B)$. Another reduction can come from a lower rank of the matrix A.

Remark 2.3. The procedure of permuting columns and applying linear transformations to the rows of the system (1) has been done with respect to the matrix $B$, but one can start by same right from the matrix $A$ as well. In this way we would obtain similar boundary conditions as (6), only the vectors $\Psi_{V}$ and $\Psi_{V}^{\prime}$ would be interchanged. Theorem 2.1 can be thus formulated with Equation (6) replaced by

$$
\left(\begin{array}{cc}
I^{(m)} & T \\
0 & 0
\end{array}\right) \Psi_{V}=\left(\begin{array}{cc}
S & 0 \\
-T^{*} & I^{(n-m)}
\end{array}\right) \Psi_{V}^{\prime} .
$$

For completeness' sake we add that another possible forms of Equation (6) in Theorem 2.1 are

$$
\left(\begin{array}{cc}
S & 0 \\
-T^{*} & I^{(n-m)}
\end{array}\right) \Psi_{V}+\left(\begin{array}{cc}
I^{(m)} & T \\
0 & 0
\end{array}\right) \Psi_{V}^{\prime}=0
$$

and

$$
\left(\begin{array}{cc}
I^{(m)} & T \\
0 & 0
\end{array}\right) \Psi_{V}+\left(\begin{array}{cc}
S & 0 \\
-T^{*} & I^{(n-m)}
\end{array}\right) \Psi_{V}^{\prime}=0 ;
$$

having the standardized form $A \Psi_{V}+B \Psi_{V}^{\prime}=0$, last two formulations may be sometimes more convenient than (6).

Obviously, an analogous remark applies to Equation (7).

Remark 2.4. A formulation of boundary conditions with a matrix structure singling out the regular part as in (7) has been derived in a different way by P. Kuchment [15]. Recall that in the setting analogous to ours he stated existence of an orthogonal projector $P$ in $\mathbb{C}^{n}$ with the complementary projector $Q=I d-P$ and a self-adjoint operator $L$ in $Q \mathbb{C}^{n}$ such that the boundary conditions may be written in the form

$$
\begin{gathered}
P \Psi_{V}=0 \\
Q \Psi_{V}^{\prime}+L Q \Psi_{V}=0 .
\end{gathered}
$$

Let us briefly explain how P. Kuchment's form differs from (7). When transformed into a matrix form, (13) consists of two groups of $n$ linearly dependent equations. If we then naturally extract a single group of $n$ linearly indepent ones, we arrive at a condition with a structure similar to (11), i. e. the upper right submatrix standing at $\Psi_{V}^{\prime}$ is generally a nonzero matrix $m \times(n-m)$. In other words, whilst P. Kuchment aimed to decompose the boundary conditions with respect to 
two complementary orthogonal projectors, our aim was to obtain a unique matrix form with as many vanishing terms as possible; the form (6) turned out to have a highly suitable structure for solving the problem of approximations that we are going to analyze in the rest of the paper.

To conclude this introductory section, let us summarize main advantages and disadvantages of the conditions (6) and (7). They are unique and exhibit a simple and clear correspondence between the parameters of the coupling and the entries of matrices in (6), furthermore, the matrices in (6) are relatively sparse. On the negative side, the structure of matrices in (6) depends on $\operatorname{rank}(B)$ and the vertex numbering is not fully permutable.

\section{The approximation arrangement}

We have argued above that due to a local character one can consider a single-vertex situation, i.e. star graph, when asking about the meaning of the vertex coupling. In this section we consider such a quantum graph with general matching conditions and show that the singular coupling may be understood as a limit case of certain family of graphs constructed only from edges connected by $\delta$-couplings, $\delta$-interactions, and supporting constant vector potentials.

Following the above discussion, one may consider the boundary conditions of the form (6), renaming the edges if necessary. It turns out that for notational purposes it is advantageous to adopt the following convention on a shift of the column indices of $T$ :

Convention 3.1. The lines of the matrix $T$ are indexed from 1 to $m$, the columns are indexed from $m+1$ to $n$.

Now we can proceed to the description of our approximating model. Consider a star graph with $n$ outgoing edges coupled in a general way given by the condition (7). The approximation in question looks as follows (cf. Fig.1):

- We take $n$ halflines, each parametrized by $x \in[0,+\infty)$, with the endpoints denoted as $V_{j}$, and put a $\delta$-coupling (to the edges specified below) with the parameter $v_{j}(d)$ at the point $V_{j}$ for all $j \in \hat{n}$.

- Certain pairs $V_{j}, V_{k}$ of halfline endpoints will be joined by edges of the length $2 d$, and the center of each such joining segment will be denoted as $W_{\{j, k\}}$. For each pair $\{j, k\}$, the points $V_{j}$ and $V_{k}, j \neq k$, are joined if one of the following three conditions is satisfied (keep in mind Convention 3.1):

(1) $j \in \hat{m}, k \geq m+1$, and $T_{j k} \neq 0$ (or $j \geq m+1, k \in \hat{m}$, and $T_{k j} \neq 0$ ),

(2) $j, k \in \hat{m}$ and $(\exists l \geq m+1)\left(T_{j l} \neq 0 \wedge T_{k l} \neq 0\right)$,

(3) $j, k \in \hat{m}, S_{j k} \neq 0$, and the previous condition is not satisfied.

- At each point $W_{\{j, k\}}$ we place a $\delta$ interaction with a parameter $w_{\{j, k\}}(d)$. From now on we use the following convention: the connecting edges of the length $2 d$ are considered as composed of two line segments of the length $d$, on each of them the variable runs from 0 (corresponding to the point $W_{\{j, k\}}$ ) to $d$ (corresponding to the point $V_{j}$ or $V_{k}$ ).

- On each connecting segment described above we put a vector potential which is constant on the whole line between the points $V_{j}$ and $V_{k}$. We denote the potential strength between the points $W_{\{j, k\}}$ and $V_{j}$ as $A_{(j, k)}(d)$, and between the points $W_{\{j, k\}}$ and $V_{k}$ as $A_{(k, j)}(d)$. It follows from the continuity that $A_{(k, j)}(d)=-A_{(j, k)}(d)$ for any pair $\{j, k\}$. 


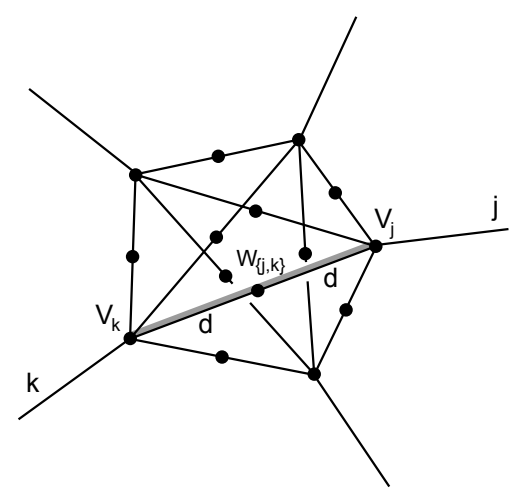

Figure 1: The scheme of the approximation. All inner links are of length $2 d$. Some connection links may be missing if the conditions given in the text are not satisfied. The quantities corresponding to the index pair $\{j, k\}$ are marked, and the grey line symbolizes the vector potential $A_{(j, k)}(d)$.

The choice of the dependence of $v_{j}(d), w_{\{j, k\}}(d)$ and $A_{(j, k)}(d)$ on the parameter $d$ is crucial for the approximation and will be specified later.

It is useful to introduce the set $N_{j} \subset \hat{n}$ containing indices of all the edges that are joined to the $j$-th one by a connecting segment, i.e.

$$
\begin{array}{rlrl}
N_{j}= & \left\{k \in \hat{m} \mid S_{j k} \neq 0\right\} \cup\{k \in \hat{m} \mid & \left.(\exists l \geq m+1)\left(T_{j l} \neq 0 \wedge T_{k l} \neq 0\right)\right\} \\
& \cup\left\{k \geq m+1 \mid T_{j k} \neq 0\right\} & & \text { for } j \in \hat{m} \\
N_{j}= & \left\{k \in \hat{m} \mid T_{k j} \neq 0\right\} & & \text { for } j \geq m+1
\end{array}
$$

The definition of $N_{j}$ has these two trivial consequences, namely

$$
\begin{gathered}
k \in N_{j} \Leftrightarrow j \in N_{k} \\
j \geq m+1 \Rightarrow N_{j} \subset \hat{m}
\end{gathered}
$$

For the wave function components on the edges we use the following symbols:

- the wave function on the $j$-th half line is denoted by $\psi_{j}$,

- the wave function on the line connecting points $V_{j}$ and $V_{k}$ has two components: the one on the line between $W_{\{j, k\}}$ and $V_{j}$ is denoted by $\varphi_{(j, k)}$, the one on the half between the middle and the endpoint of the $k$-th half line is denoted by $\varphi_{(k, j)}$. We remind once more the way in which the variable $x$ of $\varphi_{(j, k)}$ and $\varphi_{(k, j)}$ is considered: it grows from 0 at the point $W_{\{j, k\}}$ to $d$ at the point $V_{j}$ or $V_{k}$, respectively.

Next we describe how the $\delta$ couplings involved look like; for simplicity we will refrain from indicating in the boundary conditions the dependence of the parameters $u, v_{j}, w_{\{j, k\}}$ on the distance $d$.

The $\delta$ interaction at the edge connecting the $j$-th and $k$-th half line (of course, for $j, k \in \hat{n}$ such that $k \in N_{j}$ only) is expressed through the conditions

$$
\begin{gathered}
\varphi_{(j, k)}(0)=\varphi_{(k, j)}(0)=: \varphi_{\{j, k\}}(0), \\
\varphi_{(j, k)}^{\prime}\left(0_{+}\right)+\varphi_{(k, j)}^{\prime}\left(0_{+}\right)=w_{\{j, k\}} \varphi_{\{j, k\}}(0),
\end{gathered}
$$


the $\delta$ coupling at the endpoint of the $j$-th half line $(j \in \hat{n})$ means

$$
\begin{gathered}
\psi_{j}(0)=\varphi_{(j, k)}(d) \quad \text { for all } k \in N_{j}, \\
\psi_{j}^{\prime}(0)-\sum_{k \in N_{j}} \varphi_{(j, k)}^{\prime}(d)=v_{j} \psi_{j}(0) .
\end{gathered}
$$

Further relations which will help us to find the parameter dependence on $d$ come from Taylor expansion. Consider first the case without any added potential,

$$
\begin{aligned}
& \varphi_{(j, k)}(d)=\varphi_{\{j, k\}}(0)+d \varphi_{(j, k)}^{\prime}(0)+O\left(d^{2}\right), \\
& \varphi_{(j, k)}^{\prime}(d)=\varphi_{(j, k)}^{\prime}(0)+O(d), \quad j, k \in \hat{n} .
\end{aligned}
$$

To take the effect of added vector potentials into account, the following lemma will prove useful:

Lemma 3.2. Let us consider a line parametrized by the variable $x \in(0, L), L \in(0,+\infty) \cup\{+\infty\}$, and let $H$ denote a Hamiltonian of a particle on this line interacting with a potential $V$,

$$
H=-\frac{\mathrm{d}^{2}}{\mathrm{~d} x^{2}}+V
$$

sufficiently regular to make $H$ self-adjoint. We denote by $\psi^{s, t}$ the solution of $H \psi=k^{2} \psi$ with the boundary values $\psi^{s, t}(0)=s, \psi^{s, t^{\prime}}(0)=t$. Consider the same system with a vector potential $A$ added, again sufficiently regular; the Hamiltonian is consequently given by

$$
H_{A}=\left(-\mathrm{i} \frac{\mathrm{d}}{\mathrm{d} x}-A\right)^{2}+V
$$

Let $\psi_{A}^{s, t}$ denote the solution of $H_{A} \psi=k^{2} \psi$ with the same boundary values as before, i.e. $\psi_{A}^{s, t}(0)=$ $s, \psi_{A}^{s, t^{\prime}}(0)=t$. Then the function $\psi_{A}^{s, t}$ can be expressed as

$$
\psi_{A}^{s, t}(x)=\mathrm{e}^{\mathrm{i} \int_{0}^{x} A(z) \mathrm{d} z} \cdot \psi^{s, t}(x) \quad \text { for all } \quad x \in(0, L) .
$$

Proof. The aim is to prove that

$$
-\psi^{s, t^{\prime \prime}}+V \psi^{s, t}=k^{2} \psi^{s, t} \wedge \psi^{s, t}(0)=s \quad \wedge \quad \psi^{s, t^{\prime}}(0)=t
$$

implies

$$
\left(-\mathrm{i} \frac{\mathrm{d}}{\mathrm{d} x}-A\right)^{2}\left(\mathrm{e}^{\mathrm{i} \int_{0}^{x} A(z) \mathrm{d} z} \cdot \psi^{s, t}\right)+V \cdot \mathrm{e}^{\mathrm{i} \int_{0}^{x} A(z) \mathrm{d} z} \cdot \psi^{s, t}=k^{2} \mathrm{e}^{\mathrm{i} \int_{0}^{x} A(z) \mathrm{d} z} \cdot \psi^{s, t}
$$

and $\psi_{A}^{s, t}(0)=s, \psi_{A}^{s, t^{\prime}}(0)=t$. The last part is obvious, since the exponential factor involved is equal to one, hence it suffices to prove the displayed relation. It is straightforward that the Hamiltonian $H_{A}$ acts generally as

$$
H_{A}=-\frac{\mathrm{d}^{2}}{\mathrm{~d} x^{2}}+2 \mathrm{i} A \frac{\mathrm{d}}{\mathrm{d} x}+\mathrm{i} A^{\prime}+A^{2}+V .
$$

We substitute $\mathrm{e}^{\mathrm{i} \int_{0}^{x} A(z) \mathrm{d} z} \cdot \psi^{s, t}$ for $\psi$, obtaining

$$
\begin{aligned}
& {\left[H_{A}\left(\mathrm{e}^{\mathrm{i} \int_{0}^{x} A(z) \mathrm{d} z} \cdot \psi^{s, t}\right)\right](x)=-\frac{\mathrm{d}^{2}}{\mathrm{~d} x^{2}}\left(\mathrm{e}^{\mathrm{i} \int_{0}^{x} A(z) \mathrm{d} z} \cdot \psi^{s, t}\right)(x)+} \\
& +2 \mathrm{i} A(x) \frac{\mathrm{d}}{\mathrm{d} x}\left(\mathrm{e}^{\mathrm{i} \int_{0}^{x} A(z) \mathrm{d} z} \cdot \psi^{s, t}\right)(x)+\left(\mathrm{i} A^{\prime}(x)+A(x)^{2}+V(x)\right) \mathrm{e}^{\mathrm{i} \int_{0}^{x} A(z) \mathrm{d} z} \cdot \psi^{s, t}(x) .
\end{aligned}
$$


Now we express the derivatives applying the formula $\frac{\mathrm{d}}{\mathrm{d} x} \int_{0}^{x} A(z) \mathrm{d} z=A(x)$. Most of the terms then cancel, it remains only

$$
\left[H_{A}\left(\mathrm{e}^{\mathrm{i} \int_{0}^{x} A(z) \mathrm{d} z} \cdot \psi^{s, t}\right)\right](x)=\mathrm{e}^{\mathrm{i} \int_{0}^{x} A(z) \mathrm{d} z} \cdot\left(-\psi^{s, t^{\prime \prime}}(x)+V(x) \cdot \psi^{s, t}(x)\right) .
$$

Due to the assumption $-\psi^{s, t \prime}+V \psi^{s, t}=k^{2} \psi^{s, t}$, we have

$$
\left[H_{A}\left(\mathrm{e}^{\mathrm{i} \int_{0}^{x} A(z) \mathrm{d} z} \cdot \psi^{s, t}\right)\right](x)=k^{2} \mathrm{e}^{\mathrm{i} \int_{0}^{x} A(z) \mathrm{d} z} \cdot \psi^{s, t}(x),
$$

what we have set out to prove.

The lemma says that adding a vector potential on an edge of a quantum graph has a very simple effect of changing the phase of the wave function by the value $\int_{0}^{x} A(z) \mathrm{d} z$. We will work in this paper with the special case of constant vector potentials on the connecting segments of the lengths $2 d$, hence the phase shift will be given here as a product of the value $A$ and the length in question.

Lemma 3.2 has the following very useful consequence.

Corollary 3.3. Consider a quantum graph vertex with $n$ outgoing edges indexed by $1, \ldots, n$ and parametrized by $x \in\left(0, L_{j}\right)$. Suppose that there is a $\delta$ coupling with the parameter $\alpha$ at the vertex, and moreover, that there is a constant vector potential $A_{j}$ on the $j$-th edge for all $j \in \hat{n}$. Let $\psi_{j}$ denote the wave function component on the $j$-the edge. Then the boundary conditions acquire the form

$$
\begin{gathered}
\psi_{j}(0)=\psi_{k}(0)=: \psi(0) \quad \text { for all } \quad j, k \in \hat{n}, \\
\sum_{j=1}^{n} \psi_{j}^{\prime}(0)=\left(\alpha+\mathrm{i} \sum_{j=1}^{n} A_{j}\right) \psi(0),
\end{gathered}
$$

where $\psi_{j}(0), \psi_{j}^{\prime}(0)$, etc., stand for the one-sided (right) limits at $x=0$.

In other words the effect of the vector potentials on the boundary conditions corresponding to a "pure" $\delta$ coupling is the following:

- the continuity is not affected,

- the coupling parameter is changed from $\alpha$ to $\alpha+\mathrm{i} \sum_{j=1}^{n} A_{j}$.

Proof. Consider first the situation without any vector potentials. If $\psi_{j}^{0}, j \in \hat{n}$, denote the wave function components corresponding to this case, the boundary conditions expressing the $\delta$ coupling have the form (4), i.e.

$$
\begin{gathered}
\psi_{j}^{0}(0)=\psi_{k}^{0}(0)=: \psi^{0}(0) \quad \text { for all } \quad j, k \in \hat{n}, \\
\sum_{j=1}^{n} \psi_{j}^{0^{\prime}}(0)=\alpha \psi^{0}(0) .
\end{gathered}
$$

If there are vector potentials on the edges, $A_{j}$ on the $j$-th edge, one has in view of the previous lemma, $\psi_{j}(x)=\mathrm{e}^{\mathrm{i} A_{j} x} \psi_{j}^{0}(x)$, i.e.

$$
\begin{aligned}
\psi_{j}^{0}(x) & =\mathrm{e}^{-\mathrm{i} A_{j} x} \psi_{j}(x), \\
\psi_{j}^{0^{\prime}}(x)=\frac{\mathrm{d}}{\mathrm{d} x}\left(\mathrm{e}^{-\mathrm{i} A_{j} x} \psi_{j}(x)\right) & =\mathrm{e}^{-\mathrm{i} A_{j} x} \psi_{j}^{\prime}(x)-\mathrm{i} A_{j} \cdot \mathrm{e}^{-\mathrm{i} A_{j} x} \psi_{j}(x) .
\end{aligned}
$$


Thence we express $\psi_{j}^{0}(0)$ and $\psi_{j}^{0^{\prime}}(0)$ : they are equal to

$$
\begin{gathered}
\psi_{j}^{0}(0)=\psi_{j}(0), \\
\psi_{j}^{0^{\prime}}(0)=\psi_{j}^{\prime}(0)-\mathrm{i} A_{j} \psi_{j}(0) ;
\end{gathered}
$$

substituting them to (24) we obtain

$$
\begin{gathered}
\psi_{j}(0)=\psi_{k}(0)=: \psi(0) \quad \text { for all } \quad j, k \in \hat{n}, \\
\sum_{j=1}^{n}\left(\psi_{j}^{\prime}(0)-\mathrm{i} A_{j} \cdot \psi_{j}(0)\right)=\alpha \psi(0) .
\end{gathered}
$$

The first line expresses the continuity of the wavefunction in the vertex supporting the $\delta$ coupling in the same way as in the absence of vector potentials, whereas the second line shows how the condition for the sum of the derivatives is changed. With the continuity in mind, we may replace $\psi_{j}(0)$ by $\psi(0)$ obtaining

$$
\sum_{j=1}^{n} \psi_{j}^{\prime}(0)=\left(\alpha+\mathrm{i} \sum_{j=1}^{n} A_{j}\right) \psi(0),
$$

which finishes the proof.

Recall that approximating we are constructing supposes that constant vector potentials are added on the joining edges. If an edge of the length $2 d$ joins the endpoints of the $j$-th and $k$-th half line, there is a constant vector potential of the value $A_{(j, k)}(d)$ on the part of the length $d$ closer to the $j$-th half line and a constant vector potential of the value $A_{(k, j)}(d)=-A_{(j, k)}(d)$ on the part of the length $d$ closer to the $k$-th half line. With regard to Lemma 3.2, the impact of the added potentials consists in phase shifts by $d \cdot A_{(j, k)}(d)$ and $d \cdot A_{(k, j)}(d)$. Let us include this effect into the corresponding equations, i.e. into (19):

$$
\begin{aligned}
& \varphi_{(j, k)}(d)=\mathrm{e}^{\mathrm{i} d A_{(j, k)}\left(\varphi_{\{j, k\}}(0)+d \varphi_{(j, k)}^{\prime}(0)\right)+O\left(d^{2}\right),} \\
& \varphi_{(j, k)}^{\prime}(d)=\mathrm{e}^{\mathrm{i} d A_{(j, k)}} \varphi_{(j, k)}^{\prime}(0)+O(d), \quad j, k \in \hat{n} .
\end{aligned}
$$

The system of equations (17), (18), and (25) describes the relations between values of wave functions and their derivatives at all the vertices. Next we will eliminate the terms with the "auxiliary" functions $\varphi_{\{j, k\}}$ and express the relations between $2 n$ terms $\psi_{j}(0), \psi_{j}^{\prime}(0), j \in \hat{n}$.

We begin with the first one of the relations (25) together with the continuity requirement (18), which yields

$$
d \varphi_{(j, k)}^{\prime}(0)=\mathrm{e}^{-\mathrm{i} d A_{(j, k)}} \psi_{j}(0)-\varphi_{\{j, k\}}(0)+O\left(d^{2}\right) .
$$

The same relation holds with $j$ replaced by $k$, summing them together and using the second of the relations (17) we get

$$
\left(2+d w_{\{j, k\}}\right) \varphi_{\{j, k\}}(0)=\mathrm{e}^{-\mathrm{i} d A_{(j, k)}} \psi_{j}(0)+\mathrm{e}^{-\mathrm{i} d A_{(k, j)}} \psi_{k}(0)+O\left(d^{2}\right) .
$$

We express $\varphi_{\{j, k\}}(0)$ from here and substitute into the first of the equations (25); using at the same time the first relation of (18) we get

$$
\psi_{j}(0)=\mathrm{e}^{\mathrm{i} d A_{(j, k)}} \cdot\left(\frac{\mathrm{e}^{-\mathrm{i} d A_{(j, k)}} \psi_{j}(0)+\mathrm{e}^{-\mathrm{i} d A_{(k, j)}} \psi_{k}(0)+O\left(d^{2}\right)}{2+d \cdot w_{\{j, k\}}}+d \varphi_{(j, k)}^{\prime}(0)\right)+O\left(d^{2}\right),
$$


and considering the second of the equations (25), we have

$$
\psi_{j}(0)=\frac{\psi_{j}(0)+\mathrm{e}^{\mathrm{i} d\left(A_{(j, k)}-A_{(k, j)}\right)} \psi_{k}(0)+O\left(d^{2}\right)}{2+d \cdot w_{\{j, k\}}}+d \varphi_{(j, k)}^{\prime}(d)+O\left(d^{2}\right) .
$$

Since the values of vector potentials are supposed to have the "antisymmetry" property, $A_{(k, j)}(d)=$ $-A_{(j, k)}(d)$, we may simplify the above equation to

$$
\psi_{j}(0)=\frac{\psi_{j}(0)+\mathrm{e}^{2 \mathrm{i} d A_{(j, k)}} \psi_{k}(0)+O\left(d^{2}\right)}{2+d \cdot w_{\{j, k\}}}+d \varphi_{(j, k)}^{\prime}(d)+O\left(d^{2}\right) .
$$

Summing the last equation over $k \in N_{j}$ yields

$$
\begin{aligned}
\# N_{j} \cdot \psi_{j}(0)=\psi_{j}(0) \cdot \sum_{k \in N_{j}} \frac{1}{2+d \cdot w_{\{j, k\}}}+\sum_{k \in N_{j}} \frac{\mathrm{e}^{2 \mathrm{i} d A_{(j, k)}} \psi_{k}(0)}{2+d \cdot w_{\{j, k\}}}+d \cdot \sum_{k \in N_{j}} \varphi_{(j, k)}^{\prime}(d)+ \\
+\sum_{k \in N_{j}} \frac{O\left(d^{2}\right)}{2+d \cdot w_{\{j, k\}}}+O\left(d^{2}\right)
\end{aligned}
$$

( $\# N_{j}$ denotes the cardinality of $N_{j}$ ), and with the help of the second of the relations (18) we arrive at the final expression,

$$
\begin{aligned}
d \psi_{j}^{\prime}(0)=\left(d v_{j}+\# N_{j}-\sum_{k \in N_{j}} \frac{1}{2+d \cdot w_{\{j, k\}}}\right) \psi_{j}(0)-\sum_{k \in N_{j}} \frac{\mathrm{e}^{\mathrm{i} d\left(A_{(j, k)}-A_{(k, j)}\right)} \psi_{k}(0)}{2+d \cdot w_{\{j, k\}}} \\
+\sum_{k \in N_{j}} \frac{O\left(d^{2}\right)}{2+d \cdot w_{\{j, k\}}}+O\left(d^{2}\right) .
\end{aligned}
$$

Our objective is to choose $v_{j}(d), w_{\{j, k\}}(d)$ and $A_{(j, k)}(d)$ in such a way that in the limit $d \rightarrow 0$ the system of relations (28) with $j \in \hat{n}$ tends to the system of $n$ boundary conditions (7). The lines of (7) are of two types, let us recall:

$$
\begin{array}{rlr}
\psi_{j}^{\prime}(0)+\sum_{l=m+1}^{n} T_{j l} \psi_{l}^{\prime}(0) & =\sum_{k=1}^{m} S_{j k} \psi_{k}(0) & j \in \hat{m} \\
0 & =-\sum_{k=1}^{m} \overline{T_{k j}} \psi_{k}(0)+\psi_{j}(0) & j=m+1, \ldots, n .
\end{array}
$$

We point out here with reference to (14) that these relations may be written also with the summation indices running through the restricted sets, namely

$$
\begin{array}{rlr}
\psi_{j}^{\prime}(0)+\sum_{l \in N_{j} \mid \hat{m}} T_{j l} \psi_{l}^{\prime}(0) & =\sum_{k=1}^{m} S_{j k} \psi_{k}(0) & j \in \hat{m} \\
0 & =-\sum_{k \in N_{j}} \overline{T_{k j}} \psi_{k}(0)+\psi_{j}(0) & j=m+1, \ldots, n,
\end{array}
$$

since for any pair $j \in \hat{m}, l \in\{m+1, \cdots, n\}$ the implication $T_{j l} \neq 0 \Rightarrow l \in N_{j}$ holds, see also Eqs. (15), (16). 
When looking for a suitable dependence of $v_{j}(d), w_{\{j, k\}}(d)$ and $A_{(j, k)}(d)$ on $d$, we start with Eq. (28) in the case when $j \geq m+1$. Our aim is to find conditions under which (28) tends to (32) as $d \rightarrow 0$. It is obvious that the sufficient conditions are

$$
\begin{aligned}
& \lim _{d \rightarrow 0}\left(d v_{j}+\# N_{j}-\sum_{k \in N_{j}} \frac{1}{2+d \cdot w_{\{j, k\}}}\right) \in \mathbb{R} \backslash\{0\}, \\
& \lim _{d \rightarrow 0} \frac{1}{2+d \cdot w_{\{j, k\}}} \in \mathbb{R} \backslash\{0\} \quad \forall k \in N_{j}, \\
& \frac{\frac{\mathrm{e}^{2 \mathrm{i} d A_{(j, k)}}}{2+d \cdot w_{\{j, k\}}}}{d v_{j}+\# N_{j}-\sum_{h \in N_{j}} \frac{1}{2+d \cdot w_{\{j, h\}}}}=\overline{T_{k j}} \quad \forall k \in N_{j} .
\end{aligned}
$$

Now we proceed to the case $j \in \hat{m}$. Our approach is based on substitution of (28) into the left-hand side of (31) and a subsequent comparison of the right-hand sides. The substitution is straightforward,

$$
\begin{aligned}
\psi_{j}^{\prime}(0)+\sum_{l \in N_{j} \backslash \hat{m}} T_{j l} \cdot \psi_{l}^{\prime}(0) & =\left(v_{j}+\frac{\# N_{j}}{d}-\frac{1}{d} \sum_{h \in N_{j}} \frac{1}{2+d \cdot w_{\{j, h\}}}\right) \psi_{j}(0)-\frac{1}{d} \sum_{k \in N_{j}} \frac{\mathrm{e}^{2 \mathrm{i} d A_{(j, k)}} \psi_{k}(0)}{2+d \cdot w_{\{j, k\}}} \\
+\sum_{l \in N_{j} \backslash \hat{m}} T_{j l} & {\left[\left(v_{l}+\frac{\# N_{l}}{d}-\frac{1}{d} \sum_{h \in N_{l}} \frac{1}{2+d \cdot w_{\{l, h\}}}\right) \psi_{l}(0)-\frac{1}{d} \sum_{k \in N_{l}} \frac{\mathrm{e}^{2 \mathrm{i} d A_{(l, k)}} \psi_{k}(0)}{2+d \cdot w_{\{l, k\}}}\right] } \\
+ & O(d)+\sum_{k \in N_{j}} \frac{O(d)}{2+d \cdot w_{\{j, k\}}}+\sum_{l=m+1}^{n} T_{j l}\left(O(d)+\sum_{h \in N_{l}} \frac{O(d)}{2+d \cdot w_{\{l, h\}}}\right)
\end{aligned}
$$

then we apply two identities, which can be easily proven, namely

(i) $\sum_{k \in N_{j}} \frac{\mathrm{e}^{2 \mathrm{i} d A_{(j, k)}} \psi_{k}(0)}{2+d \cdot w_{\{j, k\}}}=\sum_{k \in N_{j} \cap \hat{m}} \frac{\mathrm{e}^{2 \mathrm{i} d A_{(j, k)}} \psi_{k}(0)}{2+d \cdot w_{\{j, k\}}}+\sum_{l \in N_{j} \backslash \hat{m}} \frac{\mathrm{e}^{2 \mathrm{i} d A_{(j, l)}} \psi_{l}(0)}{2+d \cdot w_{\{j, l\}}}$,

(ii) $\sum_{l \in N_{j} \backslash \hat{m}} T_{j l} \sum_{k \in N_{l}} \frac{\mathrm{e}^{2 \mathrm{i} d A_{(l, k)}} \psi_{k}(0)}{2+d \cdot w_{\{l, k\}}}$

$$
=\left(\sum_{l \in N_{j} \backslash \hat{m}} T_{j l} \frac{\mathrm{e}^{2 \mathrm{i} d A_{(l, j)}}}{2+d \cdot w_{\{l, j\}}}\right) \psi_{j}(0)+\sum_{k \in N_{j} \cap \hat{m}}\left(\sum_{l \in N_{k} \backslash \hat{m}} T_{j l} \frac{\mathrm{e}^{2 \mathrm{i} d A_{(l, k)}}}{2+d \cdot w_{\{l, k\}}}\right) \psi_{k}(0)
$$


and obtain

$$
\begin{gathered}
\psi_{j}^{\prime}(0)+\sum_{l \in N_{j} \backslash \hat{m}} T_{j l} \cdot \psi_{l}^{\prime}(0) \\
=\left(v_{j}+\frac{\# N_{j}}{d}-\frac{1}{d} \sum_{h \in N_{j}} \frac{1}{2+d \cdot w_{\{j, h\}}}-\frac{1}{d} \sum_{l \in N_{j} \backslash \hat{m}} T_{j l} \frac{\mathrm{e}^{2 \mathrm{i} d A_{(l, j)}}}{2+d \cdot w_{\{l, j\}}}\right) \psi_{j}(0) \\
\quad-\frac{1}{d} \sum_{k \in N_{j} \cap \hat{m}}\left(\frac{\mathrm{e}^{2 \mathrm{i} d A_{(j, k)}}}{2+d \cdot w_{\{j, k\}}}+\sum_{l \in N_{k} \backslash \hat{m}} T_{j l} \frac{\mathrm{e}^{2 \mathrm{i} d A_{(l, k)}}}{2+d \cdot w_{\{l, k\}}}\right) \psi_{k}(0) \\
+\sum_{l \in N_{j} \backslash \hat{m}}\left(-\frac{1}{d} \cdot \frac{\mathrm{e}^{2 \mathrm{i} d A_{(j, l)}}}{2+d \cdot w_{\{j, l\}}}+T_{j l}\left(v_{l}+\frac{\# N_{l}}{d}-\frac{1}{d} \sum_{h \in N_{l}} \frac{1}{2+d \cdot w_{\{l, h\}}}\right)\right) \psi_{l}(0) \\
\quad+O(d)+\sum_{k \in N_{j}} \frac{O(d)}{2+d \cdot w_{\{j, k\}}}+\sum_{k \in N_{j} \cap \hat{m}\left\{l \in N_{k} \cap N_{j} \backslash \hat{m}\right.} \frac{O(d)}{2+d \cdot w_{\{l, k\}}} .
\end{gathered}
$$

As we have announced above, the goal is to determine terms $v_{j}(d), w_{\{j, k\}}(d)$ and $A_{(j, k)}(d)$ such that if $d \rightarrow 0$, the right-hand side of (37) tends to the eight-hand side of (31) for all $j \in \hat{m}$. We observe that this will be the case provided

$$
\begin{gathered}
v_{j}+\frac{\# N_{j}}{d}-\frac{1}{d} \sum_{h \in N_{j}} \frac{1}{2+d \cdot w_{\{j, h\}}}-\frac{1}{d} \sum_{l \in N_{j}} T_{j l} \frac{\mathrm{e}^{2 \mathrm{i} d A_{(l, j)}}}{2+d \cdot w_{\{l, j\}}}=S_{j j}, \\
-\frac{1}{d} \frac{\mathrm{e}^{2 \mathrm{i} d A_{(j, k)}}}{2+d \cdot w_{\{j, k\}}}-\frac{1}{d} \sum_{l \in N_{k} \backslash \hat{m}} T_{j l} \frac{\mathrm{e}^{2 \mathrm{i} d A_{(l, k)}}}{2+d \cdot w_{\{l, k\}}}=S_{j k} \quad \forall k \in N_{j} \cap \hat{m}, \\
-\frac{1}{d} \frac{\mathrm{e}^{2 \mathrm{i} d A_{(j, i)}}}{2+d \cdot w_{\{j, l\}}}+T_{j l}\left(v_{l}+\frac{\# N_{l}}{d}-\frac{1}{d} \sum_{h \in N_{l}} \frac{1}{2+d \cdot w_{\{l, h\}}}\right)=0 \quad \forall l \in N_{j} \backslash \hat{m}, \\
\lim _{d \rightarrow 0} \frac{1}{2+d \cdot w_{\{j, k\}}} \in \mathbb{R} \quad \forall k \in N_{j}, \\
\lim _{d \rightarrow 0} \frac{1}{2+d \cdot w_{\{l, k\}}} \in \mathbb{R} \quad \forall k \in N_{j} \cap \hat{m}, l \in N_{k} \cap N_{j} \backslash \hat{m} .
\end{gathered}
$$

It is easy to see that the set of equations (40) for $j \in \hat{m}, l \in N_{j} \backslash \hat{m}$ is equivalent to the set (35) for $j \geq m+1, k \in N_{j}$. Similarly, Eq. (42) for $j \in \hat{m}, k \in N_{j} \cap \hat{m}, l \in N_{k} \cap N_{j} \backslash \hat{m}$ is a weaker set of conditions than (34) with $j \geq m+1, k \in N_{j}$. Finally, Eq. (41) reduces for $k \in N_{j} \backslash \hat{m}$ to (34), thus it suffices to consider (41) with $k \in N_{j} \cap \hat{m}$.

The procedure of determination of $v_{j}(d), w_{\{j, k\}}(d)$ and $A_{(j, k)}(d)$ will proceed in three steps, at the end we add the fourth step involving the verification of the limit conditions (33), (34), and (41) restricted to $k \in N_{j} \cap \hat{m}$.

Step I. We use Eq. (40) to find an expression for $w_{\{j, l\}}(d)$ and $A_{(j, l)}(d)$ when $j \in \hat{m}$ and $l \in N_{j} \backslash \hat{m}$. We begin with rearranging Eq. (35) into the form

$$
\frac{1}{2+d \cdot w_{\{j, l\}}}=\mathrm{e}^{-2 \mathrm{i} d A_{(j, l)}} \cdot T_{j l}\left(d v_{l}+\# N_{l}-\sum_{h \in N_{l}} \frac{1}{2+d \cdot w_{\{l, h\}}}\right) \quad \forall l \in N_{j} \backslash \hat{m} .
$$


Since all the terms except $\mathrm{e}^{-2 \mathrm{i} d A_{(j,)}}$ and $T_{j l}$ are real, we can obtain immediately a condition for $A_{(j, l)}$ : We put

$$
\mathrm{e}^{2 \mathrm{i} d A_{(j, l)}}=\left\{\begin{array}{ccc}
T_{j l} /\left\|T_{j l}\right\| & \text { if } & \operatorname{Re} T_{j l} \geq 0, \\
-T_{j l} /\left\|T_{j l}\right\| & \text { if } & \operatorname{Re} T_{j l}<0 ;
\end{array}\right.
$$

it is easy to see that such a choice ensures that the expression $\mathrm{e}^{-2 \mathrm{id} A_{(j, .)}} \cdot T_{j l}$ is always real. The vector potential strength may be then chosen as follows,

$$
A_{(j, l)}(d)=\left\{\begin{array}{lll}
\frac{1}{2 d} \arg T_{j l} & \text { if } & \operatorname{Re} T_{j l} \geq 0, \\
\frac{1}{2 d}\left(\arg T_{j l}-\pi\right) & \text { if } & \operatorname{Re} T_{j l}<0
\end{array}\right.
$$

for all $j \in \hat{m}, l \in N_{j} \backslash \hat{m}$. We remark that this choice is obviously not the only one possible. Note that in this situation, namely if $j \in \hat{m}$ and $l \in N_{j} \backslash \hat{m}$, the potentials do not depend on $d$. Taking (44) into account, Eq. (43) simplifies to

$$
\frac{1}{2+d \cdot w_{\{j, l\}}}=\left\langle T_{j l}\right\rangle \cdot\left(d v_{l}+\# N_{l}-\sum_{h \in N_{l}} \frac{1}{2+d \cdot w_{\{l, h\}}}\right) \quad \forall l \geq m+1, j \in N_{l} ;
$$

note that $j \in \hat{m} \wedge l \in N_{j} \backslash \hat{m} \Leftrightarrow l \geq m+1 \wedge j \in N_{l}$. The symbol $\langle\cdot\rangle$ here has the following meaning: if $c \in \mathbb{C}$, then

$$
\langle c\rangle=\left\{\begin{array}{ccc}
|c| & \text { if } \quad \operatorname{Re} c \geq 0 \\
-|c| & \text { if } \quad \operatorname{Re} c<0
\end{array}\right.
$$

Summing (45) over $j \in N_{l}$ we get

$$
\sum_{j \in N_{l}} \frac{1}{2+d \cdot w_{\{j, l\}}}=\sum_{j \in N_{l}}\left\langle T_{j l}\right\rangle \cdot\left(d v_{l}+\# N_{l}-\sum_{h \in N_{l}} \frac{1}{2+d \cdot w_{\{l, h\}}}\right),
$$

i.e.

$$
\left(1+\sum_{h \in N_{l}}\left\langle T_{h l}\right\rangle\right) \sum_{j \in N_{l}} \frac{1}{2+d \cdot w_{\langle j, l\}}}=\sum_{j \in N_{l}}\left\langle T_{j l}\right\rangle \cdot\left(d v_{l}+\# N_{l}\right) .
$$

We have to distinguish here two situations:

(i) If $1+\sum_{h \in N_{l}}\left\langle T_{h l}\right\rangle \neq 0$, one obtains

$$
\sum_{h \in N_{l}} \frac{1}{2+d \cdot w_{\{l, h\}}}=\frac{\sum_{h \in N_{l}}\left\langle T_{h l}\right\rangle}{1+\sum_{h \in N_{l}}\left\langle T_{h l}\right\rangle} \cdot\left(d v_{l}+\# N_{l}\right),
$$

and the substitution of the left-hand side into the right-hand side of (45) leads to the formula for $1 /\left(2+d \cdot w_{\{j, l\}}\right)$, namely

$$
\frac{1}{2+d \cdot w_{\{j, l\}}}=\left\langle T_{j l}\right\rangle \cdot \frac{d v_{l}+\# N_{l}}{1+\sum_{h \in N_{l}}\left\langle T_{h l}\right\rangle} \quad \forall j \in \hat{m}, l \in N_{j} \backslash \hat{m} .
$$

We observe that the sum in the denominator may be computed over the whole set $\hat{m}$ as well, since $h \notin \mathbb{N}_{l} \Rightarrow T_{h l}=0$, which slightly simplifies the formula,

$$
\frac{1}{2+d \cdot w_{\{j, l\}}}=\left\langle T_{j l}\right\rangle \cdot \frac{d v_{l}+\# N_{l}}{1+\sum_{h=1}^{m}\left\langle T_{h l}\right\rangle} \quad \forall j \in \hat{m}, l \in N_{j} \backslash \hat{m} .
$$


From here one can easily express $w_{\{j, l\}}$, if $v_{l}$ is known. However, it turns out that $v_{l}(d), l \geq m+1$ can be chosen almost arbitrarily, the only requirements are to keep the expression $d v_{l}+\# N_{l}$ nonzero and to satisfy (33), (34) and (41). The simplest choice possible is to define $v_{l}$ by the expression

$$
\frac{d v_{l}+\# N_{l}}{1+\sum_{h=1}^{m}\left\langle T_{h l}\right\rangle}=1
$$

which simplifies the expressions for other parameters. Here we obtain already expressions for $v_{l}$ and $w_{\{j, l\}}$ if $l \geq m+1$, viz

$$
\begin{gathered}
v_{l}(d)=\frac{1-\# N_{l}+\sum_{h=1}^{m}\left\langle T_{h l}\right\rangle}{d} \quad \forall l \geq m+1, \\
w_{\{j, l\}}(d)=\frac{1}{d}\left(-2+\frac{1}{\left\langle T_{j l}\right\rangle}\right) \quad \forall j \in \hat{m}, l \in N_{j} \backslash \hat{m} .
\end{gathered}
$$

(ii) If $1+\sum_{h \in N_{l}}\left\langle T_{h l}\right\rangle=0$, it holds necessarily $d v_{l}+\# N_{l}=0$, and consequently, $v_{l}=-\frac{\# N_{l}}{d}$. Note that this equation may be obtained from Eq. (46) by putting formally $1+\sum_{h=1}^{m}\left\langle T_{h l}\right\rangle=0$. It is easy to check that $w_{\{j, l\}}$ given by Eq. (47) satisfies (43) in the case $1+\sum_{h \in N_{l}}\left\langle T_{h l}\right\rangle=0$ as well. Summing these facts up, we conclude that Eqs. (46), (47) hold universally regardless whether $1+\sum_{h \in N_{l}}\left\langle T_{h l}\right\rangle$ equals zero or not.

We would like to stress that the freedom in the choice of $v_{l}(d)$ is a consequence of the fact mentioned in Remark 2.2, namely that the number of parameters of a vertex coupling decreases with the decreasing value of $\operatorname{rank}(B)$.

Step II. Equation (39) together with the results of Step I will be used to determine $w_{\{j, k\}}(d)$ and $A_{(j, k)}(d)$ in the case when $j \in \hat{m}$ and $k \in N_{j} \cap \hat{m}$. From (39) we have

$$
-\frac{\mathrm{e}^{2 \mathrm{i} d A_{(j, k)}}}{2+d \cdot w_{\{j, k\}}}=d \cdot S_{j k}+\sum_{l \in N_{k} \backslash \hat{m}} T_{j l} \frac{\mathrm{e}^{2 \mathrm{i} d A_{(l, k)}}}{2+d \cdot w_{\{l, k\}}}
$$

the pairs $(l, k)$ appearing in the sum are of the type examined in Step I, i.e. $\left.k \in \hat{m}, l \in N_{k} \backslash \hat{m}\right)$. Thus one may substitute from (46) and (47) to obtain

$$
-\frac{\mathrm{e}^{2 \mathrm{i} d A_{(j, k)}}}{2+d \cdot w_{\{j, k\}}}=d \cdot S_{j k}+\sum_{l \in N_{k} \backslash \hat{m}} T_{j l} \overline{T_{k l}} .
$$

We observe that the summation index may run through the whole set $\hat{n} \backslash \hat{m}$, because $l \geq m+1 \wedge l \notin$ $N_{k} \Rightarrow T_{k l}=0$. This allows one to obtain a more elegant formula. In a similar way as above, we find the expression for $A_{(j, k)}$,

$$
A_{(j, k)}(d)=\frac{1}{2 d} \arg \left(d \cdot S_{j k}+\sum_{l=m+1}^{n} T_{j l} \overline{T_{k l}}\right) \quad \text { for } \quad \operatorname{Re}\left(d \cdot S_{j k}+\sum_{l=m+1}^{n} T_{j l} \overline{T_{k l}}\right) \geq 0
$$

and

$$
A_{(j, k)}(d)=\frac{1}{2 d}\left[\arg \left(d \cdot S_{j k}+\sum_{l=m+1}^{n} T_{j l} \overline{T_{k l}}\right)-\pi\right] \quad \text { for } \quad \operatorname{Re}\left(d \cdot S_{j k}+\sum_{l=m+1}^{n} T_{j l} \overline{T_{k l}}\right)<0
$$


and for $w_{\{j, k\}}$,

$$
\frac{1}{2+d \cdot w_{\{j, k\}}}=-\left\langle d \cdot S_{j k}+\sum_{l=m+1}^{n} T_{j l} \overline{T_{k l}}\right\rangle .
$$

Step III. Substitution of the results of Steps I and II into Eq. (38) provides an expression for $v_{j}(d)$ in the case when $j \in \hat{m}$. A simple calculation gives

$$
v_{j}(d)=S_{j j}-\frac{\# N_{j}}{d}-\sum_{k=1}^{m}\left\langle S_{j k}+\frac{1}{d} \sum_{l=m+1}^{n} T_{j l} \overline{T_{k l}}\right\rangle+\frac{1}{d} \sum_{l=m+1}^{n}\left(1+\left\langle T_{j l}\right\rangle\right)\left\langle T_{j l}\right\rangle .
$$

Since $S$ is a self-adjoint matrix, the term $S_{j j}$ is real, thus the whole right-hand side is a real expression.

Step IV. Finally, we verify conditions (33), (34), and (41), the last one being restricted to $k \in$ $N_{j} \cap \hat{m}$. This step consists in trivial substitutions:

$$
\begin{array}{ll}
\text { (33): } & \lim _{d \rightarrow 0}\left(d v_{j}+\# N_{j}-\sum_{k \in N_{j}} \frac{1}{2+d \cdot w_{\{j, k\}}}\right)=\lim _{d \rightarrow 0} 1=1 \in \mathbb{R} \backslash\{0\} \quad \forall j \geq m+1, \\
\text { (34): } & \lim _{d \rightarrow 0} \frac{1}{2+d \cdot w_{\{j, k\}}}=\lim _{d \rightarrow 0}\left\langle T_{k j}\right\rangle=\left\langle T_{k j}\right\rangle \in \mathbb{R} \backslash\{0\} \quad \forall j \geq m+1, k \in N_{j}, \\
\text { (41): } & \lim _{d \rightarrow 0} \frac{1}{2+d \cdot w_{\{j, k\}}}=-\lim _{d \rightarrow 0}\left\langle d \cdot S_{j k}+\sum_{l=m+1}^{n} T_{j l} \overline{T_{k l}}\right\rangle=\left\langle\sum_{l=m+1}^{n} T_{j l} \overline{T_{k l}}\right\rangle \in \mathbb{R} \\
& \forall j \in \hat{m}, k \in N_{j} \cap \hat{m} .
\end{array}
$$

\section{The norm-resolvent convergence}

In the previous section we have shown that any vertex coupling in the center point of a star graph may be regarded as a limit of a certain family of graphs supporting nothing but $\delta$ couplings, $\delta$ interactions and constant vector potentials. The parameter values of all the $\delta$ 's and vector potentials have been derived using a method devised originally in $[3,7]$ for the case of a generalized point interaction on the line. The aim of this section is to give a clear meaning to this convergence. Specifically, we are going to show that the Hamiltonian of the approximating system converges to the Hamiltonian of the approximated system in the norm-resolvent sense, with the natural consequences for the convergence of eigenvalues, eigenfunctions, etc.

We denote the Hamiltonian of the star graph $\Gamma$ with the coupling (6) at the vertex as $H^{\mathrm{Ad}}$ (referring to the approximate $d$ system), and $H_{d}^{\mathrm{Ag}}$ will stand for the approximating family of graphs that has been constructed in the previous section. Symbols $R^{\mathrm{Ad}}\left(k^{2}\right)$ and $R_{d}^{\mathrm{Ag}}\left(k^{2}\right)$ will then denote the resolvents of $H^{\mathrm{Ad}}$ and $H_{d}^{\mathrm{Ag}}$ at the points $k^{2}$ from the resolvent set. Needless to say, the operators act on different spaces: $R^{\operatorname{Ad}}\left(k^{2}\right)$ on $L^{2}(G)$, where $G=\left(\mathbb{R}^{+}\right)^{n}$ corresponds to the star graph $\Gamma$, and $R_{d}^{\mathrm{Ag}}\left(k^{2}\right)$ on $L^{2}\left(G_{d}\right)$, where

$$
G_{d}=\left(\mathbb{R}^{+}\right)^{n} \oplus(0, d)^{\sum_{j=1}^{n} N_{j}} .
$$

Our goal is to compare these resolvents. In order to do that, we need to identify $R^{\mathrm{Ad}}\left(k^{2}\right)$ with the orthogonal sum

$$
R_{d}^{\mathrm{Ad}}\left(k^{2}\right)=R^{\mathrm{Ad}}\left(k^{2}\right) \oplus 0,
$$


where 0 is a zero operator acting on the space $L^{2}\left((0, d)^{\sum_{j=1}^{n} N_{j}}\right)$ which is removed in the limit. Then both the operators $R_{d}^{\mathrm{Ad}}\left(k^{2}\right)$ and $R_{d}^{\mathrm{Ag}}\left(k^{2}\right)$ are defined as acting on functions from the set $G_{d}$ which are vector functions with $n+\sum_{j=1}^{n} N_{j}$ components; we will index the components by the set

$$
\mathcal{I}=\hat{n} \cup\left\{(l, h) \mid l \in \hat{n}, h \in N_{l}\right\} .
$$

In this setting we are able to state now the main theorem of this section and the whole paper.

Theorem 4.1. Let $v_{j}, j \in \hat{n},, w_{\{j, k\}} j \in \hat{n}, k \in \mathbb{N}_{j}$ and $A^{(j, k)}(d)$ depend on $d$ according to (50), (46), (49), (47), (48) and (44), respectively. Then the family $H^{\mathrm{Ag}}(d)$ converges to $H_{d}^{\mathrm{Ad}}$ in the norm-resolvent sense as $d \rightarrow 0_{+}$.

Proof. We have to compare the resolvents $R_{d}^{\mathrm{Ad}}\left(k^{2}\right)$ and $R_{d}^{\mathrm{Ag}}\left(k^{2}\right)$. It is obviously sufficient to check the convergence in the Hilbert-Schmidt norm,

$$
\left\|R_{d}^{\mathrm{Ag}}\left(k^{2}\right)-R_{d}^{\mathrm{Ad}}\left(k^{2}\right)\right\|_{2} \rightarrow 0_{+} \quad \text { as } d \longrightarrow 0_{+},
$$

in other words, to show that the difference of the corresponding resolvent kernels denoted as $\mathcal{G}_{k}^{\mathrm{Ag}, d}$ and $\mathcal{G}_{k}^{\mathrm{Ad}, d}$, respectively, tends to zero in $L^{2}\left(G_{d} \oplus G_{d}\right)$. Recall that these kernels, or Green's functions, are in our case matrix functions with $\left(n+\sum_{j=1}^{n} N_{j}\right) \times\left(n+\sum_{j=1}^{n} N_{j}\right)$ entries. We will index the entries by pairs of indices taken from the set $\mathcal{I}$ (cf. (53)).

The proof is divided into three parts. In the first and the second part we will derive the resolvent kernels $\mathcal{G}_{k}^{\mathrm{Ag}, d}$ and $\mathcal{G}_{k}^{\mathrm{Ad}, d}$, respectively, in the last part we compare them and demonstrate the norm-resolvent convergence.

\section{Resolvent of the approximated Hamiltonian}

Let us construct first $\mathcal{G}_{k}^{\text {Ad }}$ for the star-graph Hamiltonian with the condition (1) at the vertex. We begin with $n$ independent halflines with Dirichlet condition at its endpoints; Green's function for each of them is well-known to be

$$
\mathcal{G}_{\mathrm{i} \kappa}(x, y)=\frac{\sinh \kappa x_{<} \mathrm{e}^{-\kappa x_{>}}}{\kappa},
$$

where $x_{<}:=\min \{x, y\}, x_{>}:=\max \{x, y\}$, and we put $\mathrm{i} \kappa=k$ assuming conventionally $\operatorname{Re} \kappa>0$. The sought Green's function is then given by Krein's formula [16, App. A],

$$
R^{\mathrm{Ad}}\left(k^{2}\right)=R^{H l}\left(k^{2}\right)+\sum_{j, l=1}^{n} \lambda_{j l}\left(k^{2}\right)\left(\phi_{l}\left(\overline{k^{2}}\right), \cdot\right)_{L^{2}\left(\left(\mathbb{R}^{+}\right)^{n}\right)} \phi_{j}\left(k^{2}\right),
$$

where $R^{H l}\left(k^{2}\right)$ acts on each half line as an integral operator with the kernel $\mathcal{G}_{\mathrm{ik}}$, and for $\phi_{j}\left(k^{2}\right)$ one can choose any elements of the deficiency subspaces of the largest common restriction; we will work with $\left(\phi_{j}\left(k^{2}\right)(\vec{x})\right)_{l}=\delta_{j l} \mathrm{e}^{-\kappa x_{j}}$ where the symbol $\vec{x}$ stands here for the vector $\left(x_{1}, \ldots, x_{n}\right) \in$ $\left(\mathbb{R}^{+}\right)^{n}$. Then we have

$R^{\operatorname{Ad}}\left(k^{2}\right)\left(\begin{array}{c}\psi_{1} \\ \vdots \\ \psi_{n}\end{array}\right)\left(\begin{array}{c}x_{1} \\ \vdots \\ x_{n}\end{array}\right)=\left(\begin{array}{c}\int_{0}^{+\infty} \mathcal{G}_{\mathrm{i} \kappa}\left(x_{1}, y_{1}\right) \psi_{1}\left(y_{1}\right) \mathrm{d} y_{1} \\ \vdots \\ \int_{0}^{+\infty} \mathcal{G}_{\mathrm{i} \kappa}\left(x_{n}, y_{n}\right) \psi_{n}\left(y_{n}\right) \mathrm{d} y_{n}\end{array}\right)+\sum_{j, l=1}^{n} \lambda_{j l}\left(k^{2}\right)\left(\mathrm{e}^{-\bar{\kappa} y_{l}}, \psi_{l}\left(y_{l}\right)\right)_{L^{2}\left(\mathbb{R}^{+}\right)}\left(\begin{array}{c}0 \\ \vdots \\ \mathrm{e}^{-\kappa x_{j}} \\ \vdots \\ 0\end{array}\right)$, 
which should be satisfied for any $\left(\psi_{1}, \ldots, \psi_{n}\right)^{T} \in \bigoplus_{j=1}^{n} L^{2}\left(\mathbb{R}^{+}\right)$. We observe that for all $j \in \hat{n}$, the $j$-th component on the right hand side depends only on the variable $x_{j}$. That is why one can consider the components as functions of one variable; we denote them as $g_{j}\left(x_{j}\right), j \in \hat{n}$, in other words,

$$
R^{\mathrm{Ad}}\left(k^{2}\right)\left(\begin{array}{c}
\psi_{1} \\
\vdots \\
\psi_{n}
\end{array}\right)\left(\begin{array}{c}
x_{1} \\
\vdots \\
x_{n}
\end{array}\right)=:\left(\begin{array}{c}
g_{1}\left(x_{1}\right) \\
\vdots \\
g_{n}\left(x_{n}\right)
\end{array}\right)
$$

The functions $g_{j}$ are therefore given explicitly by

$$
g_{j}\left(x_{j}\right)=\int_{0}^{+\infty} \mathcal{G}_{\mathrm{i} \kappa}\left(x_{j}, y\right) \psi_{j}(y) \mathrm{d} y+\sum_{l=1}^{n} \lambda_{j l}\left(k^{2}\right) \int_{0}^{+\infty} \mathrm{e}^{-\kappa y} \psi_{l}(y) \mathrm{d} y \cdot \mathrm{e}^{-\kappa x_{j}} .
$$

Since the resolvent maps the whole Hilbert space into the domain of the operator, these functions have to satisfy the boundary conditions at the vertex,

$$
\sum_{h=1}^{n} A_{j h} g_{h}(0)+\sum_{h=1}^{n} B_{j h} g_{h}^{\prime}(0)=0 \quad \text { for all } j \in \hat{n},
$$

where

$$
A=\left(\begin{array}{cc}
S & 0 \\
-T^{*} & I^{(n-m)}
\end{array}\right), \quad-B=\left(\begin{array}{cc}
I^{(m)} & T \\
0 & 0
\end{array}\right) .
$$

Using the explicit form of $\mathcal{G}_{\mathrm{i} \kappa}\left(x_{h}, y\right)$ and the equality $\left.\frac{\partial \mathcal{G}_{\kappa}\left(x_{h}, y\right)}{\partial x_{h}}\right|_{x_{h}=0}=\mathrm{e}^{-\kappa y}$, we find

$$
g_{h}(0)=\sum_{l=1}^{n} \lambda_{h l}\left(k^{2}\right) \int_{0}^{+\infty} \mathrm{e}^{-\kappa y} \psi_{l}(y) \mathrm{d} y
$$

and

$$
g_{h}^{\prime}(0)=\int_{0}^{+\infty} \mathrm{e}^{-\kappa y} \psi_{h}(y) \mathrm{d} y-\kappa \sum_{l=1}^{n} \lambda_{h l}\left(k^{2}\right) \int_{0}^{+\infty} \mathrm{e}^{-\kappa y} \psi_{l}(y) \mathrm{d} y .
$$

Substituting from these two relations into (55) we get a system of equations,

$$
\sum_{l=1}^{n} \int_{0}^{+\infty}\left(\sum_{h=1}^{n} A_{j h} \lambda_{h l}\left(k^{2}\right)+B_{j l}-\kappa \sum_{h=1}^{n} B_{j h} \lambda_{h l}\left(k^{2}\right)\right) \mathrm{e}^{-\kappa y} \psi_{l}(y) \mathrm{d} y=0
$$

with $j \in \hat{n}$. We require that the left-hand side vanishes for any $\psi_{1}, \psi_{2}, \ldots, \psi_{n}$; this yields the condition $A \Lambda+B-\kappa B \Lambda=0$. From here it is easy to find the matrix $\Lambda\left(k^{2}\right)$ : we have $(A-\kappa B) \Lambda=$ $-B$, and therefore

$$
\Lambda\left(k^{2}\right)=(A-\kappa B)^{-1}(-B) .
$$

Substituting the explicit forms of $A$ and $-B$ into the expression for $\Lambda$, we obtain

$$
\Lambda\left(k^{2}\right)=\left(\begin{array}{cc}
S+\kappa I^{(m)} & \kappa T \\
-T^{*} & I^{(n-m)}
\end{array}\right)^{-1}\left(\begin{array}{cc}
I^{(m)} & T \\
0 & 0
\end{array}\right),
$$

or explicitly

$$
\Lambda\left(k^{2}\right)=\left(\begin{array}{cc}
\left(S+\kappa I^{(m)}+\kappa T T^{*}\right)^{-1} & \left(S+\kappa I^{(m)}+\kappa T T^{*}\right)^{-1} T \\
T^{*}\left(S+\kappa I^{(m)}+\kappa T T^{*}\right)^{-1} & T^{*}\left(S+\kappa I^{(m)}+\kappa T T^{*}\right)^{-1} T
\end{array}\right)
$$


provided that $\left(S+\kappa I^{(m)}+\kappa T T^{*}\right)^{-1}$ is well defined. To check that the matrix $S+\kappa I^{(m)}+\kappa T T^{*}$ is regular, we notice that

$$
S+\kappa I^{(m)}+\kappa T T^{*}=S+\kappa\left(I^{(m)}+T T^{*}\right),
$$

where the matrix $I^{(m)}+T T^{*}$ is positive definite and thus regular, and the value $\kappa$ may be chosen arbitrarily with the only restriction $\operatorname{Re} \kappa>0$. Consequently, it suffices to choose $\operatorname{Re} \kappa$ big enough to make the matrix $\kappa\left(I^{(m)}+T T^{*}\right)$ dominate over $S$, which ensures the regularity of $S+\kappa\left(I^{(m)}+T T^{*}\right)$.

Having found the coefficients $\lambda_{j l}\left(k^{2}\right)$, we have fully determined the Green's function $\mathcal{G}_{\mathrm{i} K}^{\text {Ad }}$ of the approximated system. Recall that it is an $n \times n$ matrix-valued function the $(j, l)$-th element of which is given by

$$
\mathcal{G}_{\mathrm{i} \kappa, j l}^{\mathrm{Ad}}(x, y)=\delta_{j l} \frac{\sinh \kappa x_{<} \mathrm{e}^{-\kappa x>}}{\kappa}+\lambda_{j l}\left(k^{2}\right) \mathrm{e}^{-\kappa x} \mathrm{e}^{-\kappa y} ;
$$

we use the convention that $x$ is from the $j$-th halfline and $y$ from the $l$-th one. The kernel of the operator $R_{d}^{\mathrm{Ad}}\left(k^{2}\right)$ is according to (52) given simply by

$$
\mathcal{G}_{\mathrm{i} \kappa}^{\mathrm{Ad}, d}=\left(\begin{array}{c|c}
\mathcal{G}_{\mathrm{i} K}^{\mathrm{Ad}} & 0 \\
\hline 0 & 0
\end{array}\right),
$$

i.e. all entries of $\mathcal{G}_{\mathrm{i} \kappa}^{\mathrm{Ad}, d}$ except for those indexed by $j, l \in \hat{n}$ vanish.

\section{Resolvents of the approximating family of Hamiltonians}

Next we will pass to resolvent construction for the approximating family of operators $H_{d}^{\mathrm{Ag}}$. As a starting point we consider $n$ independent halflines and $\sum_{j=1}^{n} N_{j}$ lines of the length $d$ with constant vector potentials $A_{(j, l)}(d)$, both halflines and lines of the finite length are supposed to have Dirichlet endpoints. We know that the Green's function is $\mathcal{G}_{\mathrm{i} \kappa}(x, y)=\kappa^{-1} \sinh \kappa x_{<} \mathrm{e}^{-\kappa x_{>}}$in the case of the halflines. The Green's function in the case of the lines of the length $d$ will be found in two steps. We begin with a line without vector potential and with Dirichlet endpoints; the Green's function can be easily derived being equal to

$$
\tilde{\mathcal{G}}_{\mathrm{i} \kappa}(x, y)=\frac{\sinh \kappa x_{<} \sinh \kappa\left(d-x_{>}\right)}{\kappa \sinh \kappa d} .
$$

The Hamiltonian of a free particle on a line segment acts as $-\frac{\mathrm{d}^{2}}{\mathrm{~d} x^{2}}$, if a vector potential $A$ is added it changes to $\left(-\mathrm{i} \frac{\mathrm{d}}{\mathrm{d} x}-A\right)^{2}$. Using Lemma 3.3 it is easy to check that

$$
\left(-\mathrm{i} \frac{\mathrm{d}}{\mathrm{d} x}-A\right)^{2}=U\left(-\frac{\mathrm{d}^{2}}{\mathrm{~d} x^{2}}\right) U^{*}
$$

where $U$ is the unitary operator acting as

$$
(U \psi)(x)=\mathrm{e}^{\mathrm{i} A x} \psi(x) .
$$

If we denote $H_{0}=-\frac{\mathrm{d}^{2}}{\mathrm{~d} x^{2}}$ and $H_{A}=\left(-\mathrm{i} \frac{\mathrm{d}}{\mathrm{d} x}-A\right)^{2}$, we see that

$$
\left(H_{A}-\lambda\right)^{-1}=\left(U H_{0} U^{*}-\lambda\right)^{-1}=\left(U\left(H_{0}-\lambda\right) U^{*}\right)^{-1}=U\left(H_{0}-\lambda\right)^{-1} U^{*},
$$


so the corresponding resolvents are related by the relation analogous to (59). This yields

$$
\begin{aligned}
\left(\left(H_{A}-\lambda\right)^{-1} \psi\right)(x)=\left(U\left(H_{A}-\lambda\right)^{-1} U^{*} \psi\right)(x) & =\mathrm{e}^{\mathrm{i} A x} \int_{0}^{d} \tilde{\mathcal{G}}_{\mathrm{i} \kappa}(x, y) \mathrm{e}^{-\mathrm{i} A y} \psi(y) \mathrm{d} y \\
& =\int_{0}^{d} \mathrm{e}^{\mathrm{i} A x} \frac{\sinh \kappa x_{<} \sinh \kappa\left(d-x_{>}\right)}{\kappa \sinh \kappa d} \mathrm{e}^{-\mathrm{i} A y} \psi(y) \mathrm{d} y,
\end{aligned}
$$

thus the sought integral kernel is equal to

$$
\tilde{\mathcal{G}}_{\mathrm{i} \kappa}^{A}(x, y)=\mathrm{e}^{\mathrm{i} A x} \frac{\sinh \kappa x_{<} \sinh \kappa\left(d-x_{>}\right)}{\kappa \sinh \kappa d} \mathrm{e}^{-\mathrm{i} A y} .
$$

Now we can proceed to the derivation of the complete resolvent $R_{d}^{\mathrm{Ag}}\left(k^{2}\right)$ which will be done again by means of the Krein's formula. The situation here is more complicated than in the case of the approximated system; recall that $R_{d}^{\mathrm{Ag}}\left(k^{2}\right)$, as well as $H_{d}^{\mathrm{Ad}}\left(k^{2}\right)$, acts on the larger Hilbert space $L^{2}\left(G_{d}\right)$, where $G_{d}$ has been defined in (51). Moreover, the application of Krein's formula means that we have to connect all the line segments using the appropriate boundary conditions, i.e. we must change boundary conditions at $n+2 \sum_{j=1}^{n} N_{j}$ endpoints, specifically $n$ belonging to $n$ half lines and $2 \sum_{j=1}^{n} N_{j}$ belonging to $\sum_{j=1}^{n} N_{j}$ segments of the length $d$. Thus the index set for the indices in the sum on the right hand side of the formula has $n+2 \sum_{j=1}^{n} N_{j}$ elements; we will index them by the set

$$
\hat{\mathcal{I}}=\hat{n} \cup\left\{(l, h)^{0} \mid l \in \hat{n}, h \in N_{l}\right\} \cup\left\{(l, h)^{d} \mid l \in \hat{n}, h \in N_{l}\right\} .
$$

The elements of $\hat{n}$ correspond to changed boundary conditions at the endpoints of the half lines, and the elements of the type $(l, h)^{0}$ and $(l, h)^{d}\left(h \in N_{l}\right)$ correspond to changed boundary conditions at the endpoints of the segments of the length $d$ which are connected to the endpoint of the $l$-th half line. If we denote by the symbol $R_{d}^{\mathrm{Dc}}\left(k^{2}\right)$ the resolvent of the system of the $n+\sum_{j=1}^{n} N_{j}$ decomposed edges with Dirichlet boundary conditions at the endpoints, Krein's formula for this pair of operators has the form

$$
R_{d}^{\mathrm{Ag}}\left(k^{2}\right)=R_{d}^{\mathrm{Dc}}\left(k^{2}\right)+\sum_{J, L \in \hat{\mathcal{I}}} \lambda_{J L}^{d}\left(k^{2}\right)\left(\phi_{L}^{d}\left(\overline{k^{2}}\right), \cdot\right)_{L^{2}\left(G_{d}\right)} \phi_{J}^{d}\left(k^{2}\right)
$$

The role of the superscript $d$ in the lambda symbols is to distinguish them from $\lambda_{j l}$ that have been used in Eq. (54) for the resolvent of the approximated system. The functions $\phi_{J}^{d}(J \in \hat{\mathcal{I}})$ may be chosen, as before in the case of the approximated system, as any elements of the corresponding deficiency subspaces of the largest common restriction. Note that each function $\phi_{J}^{d}$ has $n+\sum_{j=1}^{n} N_{j}$ components indexed by elements of the set $\mathcal{I}=\hat{n} \cup\left\{(l, h) \mid l \in \hat{n}, h \in N_{l}\right\}$. It turns out that a suitable choice is

$$
\begin{aligned}
\left(\phi_{j}\left(k^{2}\right)^{d}(\vec{x})\right)_{\tilde{L}} & =\delta_{j \tilde{L}} \mathrm{e}^{-\kappa x_{j}} & & \text { for } \quad j \in \hat{n}, \tilde{L} \in \mathcal{I}, \\
\left(\phi_{(l, h)^{0}}^{d}\left(k^{2}\right)(\vec{x})\right)_{\tilde{L}} & =\mathrm{e}^{\mathrm{i} A_{(l, h)} x_{(l, h)}} \delta_{(l, h) \tilde{L}} \sinh \kappa x_{(l, h)} & & \text { for } \quad l \in \hat{n}, h \in N_{l}, \tilde{L} \in \mathcal{I} \\
\left(\phi_{(l, h)^{d}}^{d}\left(k^{2}\right)(\vec{x})\right)_{\tilde{L}} & =\mathrm{e}^{\left.\mathrm{i} A_{(l, h)} x_{(l, h)}\right)} \delta_{(l, h) \tilde{L}} \sinh \kappa\left(d-x_{(l, h)}\right) & & \text { for } \quad l \in \hat{n}, h \in N_{l}, \tilde{L} \in \mathcal{I},
\end{aligned}
$$

where the symbol $\vec{x}$ denotes the vector from $G_{d}$ with the components indexed by $\mathcal{I}$. We remark that if $J \in \hat{n}, \phi_{J}^{d}$ is independent of $d$ and equal to the corresponding function chosen above in the case of the approximated system. 
If we apply the operator (60) to an arbitrary $\Psi \in \bigoplus_{j=1}^{n} L^{2}\left(G_{d}\right)$, we obtain a vector function with $n+\sum_{j=1}^{n} N_{j}$ components indexed by $\mathcal{I}$, we denote them by $g_{j}(j \in \hat{n})$ and $g_{(l, h)}$ with $l \in \hat{n}, h \in$ $N_{l}$. As in the case of the approximated system, a component $g_{J}$ depends on $x_{J}$ only, thus each $g_{J}$ can be considered as a function of a single variable. A calculation leads to the following explicit expressions for $g_{j}, j \in \hat{n}$ and $g_{(l, h)}, l \in \hat{n}, h \in N_{l}$; for better clarity we distinguish the integral variables on $\mathbb{R}^{+}$and on $(0, d)$ by a tilde, i.e. $y \in \mathbb{R}^{+}, \tilde{y} \in(0, d)$.

$$
\begin{aligned}
& g_{j}\left(x_{j}\right)=\int_{0}^{+\infty} \mathcal{G}_{\mathrm{i} \kappa}\left(x_{j}, y\right) \psi_{j}(y) \mathrm{d} y+\sum_{j^{\prime}=1}^{n} \lambda_{j j^{\prime}}^{d}\left(k^{2}\right) \int_{0}^{+\infty} \mathrm{e}^{-\kappa y} \cdot \psi_{j^{\prime}}(y) \mathrm{d} y \cdot \mathrm{e}^{-\kappa x_{j}} \\
& +\sum_{l^{\prime}=1}^{n} \sum_{h^{\prime} \in N_{l^{\prime}}}\left(\lambda_{\left.j\left(l^{\prime} h^{\prime}\right)^{0}\right)}^{d}\left(k^{2}\right) \int_{0}^{d} \mathrm{e}^{-\mathrm{i} A_{\left(l^{\prime}, h^{\prime}\right)} \tilde{y}} \sinh \kappa \tilde{y} \cdot \psi_{\left(l^{\prime}, h^{\prime}\right)}(\tilde{y}) \mathrm{d} \tilde{y}\right. \\
& \left.+\lambda_{j\left(l^{\prime} h^{\prime}\right)^{d}}^{d}\left(k^{2}\right) \int_{0}^{d} \mathrm{e}^{-\mathrm{i} A_{\left(l^{\prime}, h^{\prime}\right)} \tilde{y}} \sinh \kappa(d-\tilde{y}) \cdot \psi_{\left(l^{\prime}, h^{\prime}\right)}(\tilde{y}) \mathrm{d} \tilde{y}\right) \cdot \mathrm{e}^{-\kappa x_{j}} . \\
& g_{(l, h)}\left(x_{(l, h)}\right)=\int_{0}^{d} \tilde{\mathcal{G}}_{\mathrm{i} \kappa}^{A_{(l, h)}}\left(x_{(l, h)}, \tilde{y}\right) \psi_{(l, h)}(\tilde{y}) \mathrm{d} \tilde{y} \\
& +\mathrm{e}^{\mathrm{i} A_{(l, h)} x_{(l, h)}} \cdot \sinh \kappa x_{(l, h)} \cdot\left[\sum_{j^{\prime}=1}^{n} \lambda_{(l, h)^{0} j^{\prime}}^{d}\left(k^{2}\right) \int_{0}^{+\infty} \mathrm{e}^{-\kappa y} \cdot \psi_{j^{\prime}}(y) \mathrm{d} y\right. \\
& +\sum_{l^{\prime}=1}^{n} \sum_{h^{\prime} \in N_{l^{\prime}}}\left(\lambda_{(l, h)^{0}\left(l^{\prime} h^{\prime}\right)^{0}}^{d}\left(k^{2}\right) \int_{0}^{d} \mathrm{e}^{-\mathrm{i} A_{\left(l^{\prime}, h^{\prime}\right.} \tilde{y}} \sinh \kappa \tilde{y} \cdot \psi_{\left(l^{\prime}, h^{\prime}\right)}(\tilde{y}) \mathrm{d} \tilde{y}\right. \\
& \left.\left.+\lambda_{(l, h)^{0}\left(l^{\prime} h^{\prime}\right)^{d}}^{d}\left(k^{2}\right) \int_{0}^{d} \mathrm{e}^{-\mathrm{i} A_{\left(l^{\prime}, h^{\prime}\right)} \tilde{y}} \sinh \kappa(d-\tilde{y}) \cdot \psi_{\left(l^{\prime}, h^{\prime}\right)}(\tilde{y}) \mathrm{d} \tilde{y}\right)\right] \\
& +\mathrm{e}^{\mathrm{i} A_{(l, h)} x_{(l, h)}} \cdot \sinh \kappa\left(d-x_{(l, h)}\right) \cdot\left[\sum_{j^{\prime}=1}^{n} \lambda_{(l, h)^{d} j^{\prime}}^{d}\left(k^{2}\right) \int_{0}^{+\infty} \mathrm{e}^{-\kappa y} \cdot \psi_{j^{\prime}}(y) \mathrm{d} y\right. \\
& +\sum_{l^{\prime}=1}^{n} \sum_{h^{\prime} \in N_{l^{\prime}}}\left(\lambda_{(l, h)^{d}\left(l^{\prime} h^{\prime}\right)^{0}}^{d}\left(k^{2}\right) \int_{0}^{d} \mathrm{e}^{-\mathrm{i} A_{\left(l^{\prime}, h^{\prime}\right.} \tilde{y}} \sinh \kappa \tilde{y} \cdot \psi_{\left(l^{\prime}, h^{\prime}\right)}(\tilde{y}) \mathrm{d} \tilde{y}\right. \\
& \left.\left.+\lambda_{(l, h)^{d}\left(l^{\prime} h^{\prime}\right)^{d}}^{d}\left(k^{2}\right) \int_{0}^{d} \mathrm{e}^{-\mathrm{i} A_{\left(l^{\prime}, h^{\prime}\right)} \tilde{y}} \sinh \kappa(d-\tilde{y}) \cdot \psi_{\left(l^{\prime}, h^{\prime}\right)}(\tilde{y}) \mathrm{d} \tilde{y}\right)\right] .
\end{aligned}
$$

By definition the function $\left(g_{J}\right)_{J \in I}$ belongs to the domain of the operator $H_{d}^{\mathrm{Ag}}$, in particular, it has to satisfy the boundary conditions at the points where the edges are connected by $\delta$ interactions and $\delta$ couplings. Step by step we will write down now all these boundary conditions; this will lead to the explicit expressions for the coefficients $\lambda_{J L}^{d}\left(k^{2}\right)$.

Step 1 . The continuity at the points $W_{\{j, k\}}$ means

$$
g_{(l, h)}(0)=g_{(h, l)}(0)
$$


for all $l \in \hat{n}, h \in N_{l}$. Since $\tilde{\mathcal{G}}_{\mathrm{i} \kappa}^{A_{(, h)}}(0, \tilde{y})=0$ for all $\tilde{y} \in(0, d)$, it holds

$$
\begin{aligned}
g_{(l, h)}(0)=\sinh \kappa d & \cdot\left[\sum_{j^{\prime}=1}^{n} \lambda_{(l, h)^{d} j^{\prime}}^{d}\left(k^{2}\right) \int_{0}^{+\infty} \mathrm{e}^{-\kappa y} \psi_{j^{\prime}}(y) \mathrm{d} y\right. \\
& +\sum_{l^{\prime}=1}^{n} \sum_{h^{\prime} \in N_{l^{\prime}}}\left(\lambda_{\left.(l, h)^{d}\left(l^{\prime} h^{\prime}\right)^{0}\right)}^{d}\left(k^{2}\right) \int_{0}^{d} \mathrm{e}^{-\mathrm{i} A_{\left(l^{\prime}, h^{\prime}\right)} \tilde{y}} \sinh \kappa \tilde{y} \psi_{\left(l^{\prime}, h^{\prime}\right)}(\tilde{y}) \mathrm{d} \tilde{y}\right. \\
& \left.\left.+\lambda_{(l, h)^{d}\left(l^{\prime} h^{\prime}\right)^{d}}^{d}\left(k^{2}\right) \int_{0}^{d} \mathrm{e}^{-\mathrm{i} A_{\left(l^{\prime}, h^{\prime}\right)} \tilde{y}} \sinh \kappa(d-\tilde{y}) \psi_{\left(l^{\prime}, h^{\prime}\right)}(\tilde{y}) \mathrm{d} \tilde{y}\right)\right],
\end{aligned}
$$

the expression for $g_{(h, l)}(0)$ is similar, just the positions of $l$ and $h$ are interchanged. Since Eq. (63) must be satisfied for any choice of the function $\Psi=\left(\psi_{J}\right)_{J \in I}$, the following equalities obviously hold for $\forall l \in \hat{n}, h \in N_{l}$ :

$$
\begin{aligned}
\lambda_{(l, h)^{d} j^{\prime}}^{d}\left(k^{2}\right) & =\lambda_{(h, l) j^{d}}^{d}\left(k^{2}\right) & & \forall j^{\prime} \in \hat{n}, \\
\lambda_{(l, h)^{d}\left(l^{\prime}, h^{\prime}\right){ }^{0}}^{d}\left(k^{2}\right) & =\lambda_{(h, l)^{d}\left(l^{\prime}, h^{\prime}\right)^{0}}^{d}\left(k^{2}\right) & & \forall l^{\prime} \in \hat{n}, h^{\prime} \in N_{l^{\prime}}, \\
\lambda_{(l, h)^{d}\left(l^{\prime}, h^{\prime}\right)^{d}}^{d}\left(k^{2}\right) & =\lambda_{(h, l)^{d}\left(l^{\prime}, h^{\prime}\right)^{d}}^{d}\left(k^{2}\right) & & \forall l^{\prime} \in \hat{n}, h^{\prime} \in N_{l^{\prime}} .
\end{aligned}
$$

In other words, all the coefficients $\lambda_{(l, h)^{d} J}^{d}\left(k^{2}\right)$ with $J \in \hat{\mathcal{I}}\left(k^{2}\right)$ are symmetric with respect to an interchange of $l$ and $h$.

Step 2. The sum of derivatives in points $W_{\{j, k\}}$ is

$$
g_{(l, h)}^{\prime}(0)+g_{(h, l)}^{\prime}(0)=w_{\{l, h\}} \cdot g_{(l, h)}(0)
$$

for all $l \in \hat{n}, h \in N_{l}$. We substitute

and

$$
\begin{gathered}
\left.\frac{\partial \tilde{\mathcal{G}}_{\mathrm{i} \kappa}^{A}(x, \tilde{y})}{\partial x}\right|_{x=0}=\frac{\sinh \kappa(d-\tilde{y})}{\sinh \kappa d} \mathrm{e}^{-\mathrm{i} A \tilde{y}}, \\
\left.\left(\mathrm{e}^{\mathrm{i} A x} \sinh \kappa x\right)^{\prime}\right|_{x=0}=\kappa
\end{gathered}
$$

$$
\left.\left(\mathrm{e}^{\mathrm{i} A x} \sinh \kappa(d-x)\right)^{\prime}\right|_{x=0}=\kappa \cosh \kappa d-\mathrm{i} A \sinh \kappa d
$$

into Eq. (65) and require the equality to be satisfied for any $\Psi=\left(\psi_{J}\right)_{J \in I}$. In the course of the calculation, the outcome of the Step 1 is also used. As a result, we find how the coefficients $\lambda_{(l, h)^{d} J}^{d}\left(k^{2}\right)(J \in \mathcal{I})$ can be expressed in terms of $\lambda_{(l, h)^{0} J}^{d}\left(k^{2}\right)$ and $\lambda_{(h, l)^{0} J}^{d}\left(k^{2}\right)$ :

$$
\begin{gathered}
\lambda_{(l, h)^{d} j^{\prime}}^{d}\left(k^{2}\right)=\frac{\kappa}{2 \kappa \cosh \kappa d+w_{\{l, h\}} \sinh \kappa d}\left(\lambda_{(l, h)^{0} j^{\prime}}^{d}\left(k^{2}\right)+\lambda_{\left(h, l^{0} j^{\prime}\right.}^{d}\left(k^{2}\right)\right) \quad \forall j^{\prime} \in \hat{n}, \\
\lambda_{(l, h)^{d}\left(l^{\prime}, h^{\prime}\right)^{0}}^{d}\left(k^{2}\right)=\frac{\kappa}{2 \kappa \cosh \kappa d+w_{\{l, h\}} \sinh \kappa d}\left(\lambda_{(l, h)^{0}\left(l^{\prime}, h^{\prime}\right)^{0}}^{d}\left(k^{2}\right)+\lambda_{(h,)^{0}\left(l^{\prime}, h^{\prime}\right)^{0}}^{d}\left(k^{2}\right)\right) \quad \forall l^{\prime} \in \hat{n}, h^{\prime} \in N_{l^{\prime}}, \\
\lambda_{(l, h)^{d}\left(l^{\prime}, h^{\prime}\right)^{d}}^{d}\left(k^{2}\right)=\frac{\kappa}{2 \kappa \cosh \kappa d+w_{\{l, h\}} \sinh \kappa d}\left(\lambda_{(l, h)^{0}\left(l^{\prime}, h^{\prime}\right)^{d}}^{d}\left(k^{2}\right)+\lambda_{\left(h, l^{0}\left(l^{\prime}, h^{\prime}\right)^{d}\right.}^{d}\left(k^{2}\right)+\frac{\delta_{(l, h)\left(l^{\prime}, h^{\prime}\right)}}{\kappa \sinh \kappa d}\right) \\
\forall l^{\prime} \in \hat{n}, h^{\prime} \in N_{l^{\prime}}
\end{gathered}
$$


for the indices $l \in \hat{n}, h \in N_{l}$.

Step 3 . The continuity at the points $V_{j}$ requires

$$
g_{j}(0)=g_{(j, h)}(d)
$$

for all $j \in \hat{n}, h \in N_{j}$. Since $\mathcal{G}_{\mathrm{i} \kappa}(0, y)=0$ for all $x \in \mathbb{R}^{+}$and $\tilde{\mathcal{G}}_{\mathrm{i} \kappa}^{A_{(l, h)}}(d)=0$ for all $\tilde{y} \in(0, d)$, it holds

$$
\begin{aligned}
g_{j}(0)=\sum_{j^{\prime}=1}^{n} \lambda_{j j^{\prime}}^{d}\left(k^{2}\right) & \int_{0}^{+\infty} \mathrm{e}^{-\kappa y} \psi_{j^{\prime}}(y) \mathrm{d} y \cdot \mathrm{e}^{-\kappa x_{j}} \\
& +\sum_{l^{\prime}=1}^{n} \sum_{h^{\prime} \in N_{l^{\prime}}}\left(\lambda_{j\left(l^{\prime} h^{\prime}\right)^{0}}^{d}\left(k^{2}\right) \int_{0}^{d} \mathrm{e}^{-\mathrm{i} A_{\left(l^{\prime}, h^{\prime}, \tilde{y}\right.}} \sinh \kappa \tilde{y} \psi_{\left(l^{\prime}, h^{\prime}\right)}\right)(\tilde{y}) \mathrm{d} \tilde{y} \\
& \left.\quad+\lambda_{j\left(l^{\prime} h^{\prime}\right)^{d}}^{d}\left(k^{2}\right) \int_{0}^{d} \mathrm{e}^{-\mathrm{i} A_{\left(l^{\prime}, h^{\prime},\right.} \tilde{y}} \sinh \kappa(d-\tilde{y}) \psi_{\left(l^{\prime}, h^{\prime}\right)}(\tilde{y}) \mathrm{d} \tilde{y}\right)
\end{aligned}
$$

and

$$
\begin{aligned}
g_{(j, h)}(d)=\mathrm{e}^{\mathrm{i} A_{(j, h)} d} \cdot \sinh \kappa d \cdot\left[\sum_{j^{\prime}=1}^{n} \lambda_{(j, h)^{0} j^{\prime}}^{d}\left(k^{2}\right) \int_{0}^{+\infty} \mathrm{e}^{-\kappa y} \psi_{j^{\prime}}(y) \mathrm{d} y\right. \\
+\sum_{l^{\prime}=1}^{n} \sum_{h^{\prime} \in N_{l^{\prime}}}\left(\lambda_{(j, h)^{0}\left(l^{\prime} h^{\prime}\right)^{0}}^{d}\left(k^{2}\right) \int_{0}^{d} \mathrm{e}^{-\mathrm{i} A_{\left(l^{\prime}, h^{\prime}\right)^{\prime}} \tilde{y}} \sinh \kappa \tilde{y} \psi_{\left(l^{\prime}, h^{\prime}\right)}(\tilde{y}) \mathrm{d} \tilde{y}\right. \\
\left.\left.\quad+\lambda_{(j, h)^{0}\left(l^{\prime} h^{\prime}\right)^{d}}^{d}\left(k^{2}\right) \int_{0}^{d} \mathrm{e}^{-\mathrm{i} A_{\left(l^{\prime}, h^{\prime}\right.} \tilde{y}} \sinh \kappa(d-\tilde{y}) \psi_{\left(l^{\prime}, h^{\prime}\right)}(\tilde{y}) \mathrm{d} \tilde{y}\right)\right] .
\end{aligned}
$$

The relation (67) should be satisfied for any choice of $\Psi=\left(\psi_{J}\right)_{J \in I}$, hence we obtain the coefficients $\lambda_{(j, h)^{0} J}^{d}\left(k^{2}\right)$ with $J \in I$ expressed in terms of $\lambda_{j J}^{d}\left(k^{2}\right)$ in the following way

$$
\begin{array}{rrr}
\lambda_{(j, h)^{0} j^{\prime}}^{d}\left(k^{2}\right)=\frac{1}{\sinh \kappa d} \mathrm{e}^{-\mathrm{i} d A_{(j, h)}} \cdot \lambda_{j j^{\prime}}^{d}\left(k^{2}\right) & \forall j^{\prime} \in \hat{n}, \\
\lambda_{\left.\left(j, h^{0}\right)^{0}\left(l^{\prime}, h^{\prime}\right)^{0}\right)}^{d}\left(k^{2}\right)=\frac{1}{\sinh \kappa d} \mathrm{e}^{-\mathrm{i} d A_{(j, h)}} \cdot \lambda_{j\left(l^{\prime}, h^{\prime}\right)^{0}}^{d}\left(k^{2}\right) & \forall l^{\prime} \in \hat{n}, h^{\prime} \in N_{l^{\prime}}, \\
\lambda_{(j, h)^{0}\left(l^{\prime}, h^{\prime}\right)^{d} d}^{d}\left(k^{2}\right)=\frac{1}{\sinh \kappa d} \mathrm{e}^{-\mathrm{i} d A_{(j, h)}} \cdot \lambda_{j\left(l^{\prime}, h^{\prime}\right)^{d}}^{d}\left(k^{2}\right) & \forall l^{\prime} \in \hat{n}, h^{\prime} \in N_{l^{\prime}}
\end{array}
$$

for $j \in \hat{n}, h \in N_{j}$. We also return to the result of Step 2 - we substitute there for $\lambda_{(j, h)^{0} J}^{d}\left(k^{2}\right)$ the expressions that we have just obtained arriving thus at

$$
\begin{aligned}
& \lambda_{(l, h)^{d} j^{\prime}}^{d}\left(k^{2}\right)=\frac{\kappa}{2 \kappa \cosh \kappa d+w_{\{l, h\}} \sinh \kappa d} \cdot \frac{1}{\sinh \kappa d} \cdot\left(\mathrm{e}^{-\mathrm{i} d A_{(l, h)}} \lambda_{l j^{\prime}}^{d}\left(k^{2}\right)+\mathrm{e}^{\left.-\mathrm{i} d A_{(h, l)}\right)} \lambda_{h j^{\prime}}^{d}\left(k^{2}\right)\right) \quad \forall j^{\prime} \in \hat{n}, \\
& \lambda_{(l, h)^{d}\left(l^{\prime}, h^{\prime}\right)^{0}}^{d}\left(k^{2}\right)=\frac{\kappa}{2 \kappa \cosh \kappa d+w_{\{l, h\}} \sinh \kappa d} \cdot \frac{1}{\sinh \kappa d} \cdot\left(\mathrm{e}^{-\mathrm{i} d A_{(l, h)}} \lambda_{l\left(l^{\prime}, h^{\prime}\right)^{0}}^{d}\left(k^{2}\right)+\mathrm{e}^{-\mathrm{i} d A_{(h, l)}} \lambda_{h\left(l^{\prime}, h^{\prime}\right)^{0}}^{d}\left(k^{2}\right)\right) \\
& \forall l^{\prime} \in \hat{n}, h^{\prime} \in N_{l^{\prime}},
\end{aligned}
$$




$$
\begin{aligned}
& \lambda_{(l, h)^{d}\left(l^{\prime}, h^{\prime}\right)^{d}}^{d}\left(k^{2}\right)= \frac{\kappa}{2 \kappa \cosh \kappa d+w_{\{l, h\}} \sinh \kappa d} \cdot \frac{1}{\sinh \kappa d} . \\
& \cdot\left(\mathrm{e}^{-\mathrm{i} d A_{(l, h)}} \cdot \lambda_{l\left(l^{\prime}, h^{\prime}\right)^{d}}^{d}\left(k^{2}\right)+\mathrm{e}^{-\mathrm{i} d A_{(h, l)}} \cdot \lambda_{h\left(l^{\prime}, h^{\prime}\right)^{d}}^{d}\left(k^{2}\right)+\frac{1}{\kappa} \delta_{(l, h)\left(l^{\prime}, h^{\prime}\right)}\right) \\
& \forall l^{\prime} \in \hat{n}, h^{\prime} \in N_{l^{\prime}}
\end{aligned}
$$

for $l \in \hat{n}, h \in N_{l}$.

Step 4. In this step we examine the sum of derivatives at the points $V_{j}$, i.e. at the junctions of the halflines and connecting segments. Since the connecting lines support constant vector potentials, one has to rewrite the original condition into the form derived in Corollary 3.3. Note that the variable on the connecting segments is considered in the ingoing sense, thus the sign of the potentials $A_{(j, h)}\left(h \in N_{j}\right)$ has to be taken with the minus sign. The resulting condition is

$$
g_{j}^{\prime}(0)-\sum_{h \in N_{j}} g_{(j, h)}^{\prime}(d)=\left(v_{j}-\mathrm{i} \sum_{h \in N_{j}} A_{(j, h)}\right) \cdot g_{j}(0)
$$

for all $j \in \hat{n}$.

The way how to proceed in this step is essentially the same as in previous steps, only the calculus is slightly longer. With the aid of the formulæ

$$
\begin{gathered}
\left.\frac{\partial \mathcal{G}_{\mathrm{i} \kappa}(x, y)}{\partial x}\right|_{x=0}=\mathrm{e}^{-\kappa y}, \\
\left.\frac{\partial \tilde{\mathcal{G}}_{\mathrm{i} \kappa}^{A}(x, \tilde{y})}{\partial x}\right|_{x=d}=-\frac{\mathrm{e}^{\mathrm{i} A d}}{\sinh \kappa d} \cdot \sinh \kappa \tilde{y} \mathrm{e}^{-\mathrm{i} A \tilde{y}}
\end{gathered}
$$

and

$$
\begin{gathered}
\left.\left(\mathrm{e}^{\mathrm{i} A x} \sinh \kappa x\right)^{\prime}\right|_{x=d}=(\kappa \cosh \kappa d+\mathrm{i} A \sinh \kappa d) \cdot \mathrm{e}^{\mathrm{i} A d}, \\
\left.\left(\mathrm{e}^{\mathrm{i} A x} \sinh \kappa(d-x)\right)^{\prime}\right|_{x=d}=-\kappa \mathrm{e}^{\mathrm{i} A d},
\end{gathered}
$$

used in Eq. (72), we arrive at an expression containing $\psi_{1} \ldots, \psi_{n}$ that should be satisfied for any choice of $\Psi=\left(\psi_{J}\right)_{J \in \mathcal{I}}$. This yields the following three groups of conditions:

$$
\begin{array}{r}
\delta_{j j^{\prime}}-\kappa \lambda_{j j^{\prime}}^{d}\left(k^{2}\right)-\sum_{h \in N_{j}}\left(\kappa \cosh \kappa d+\mathrm{i} A_{(j, h)} \sinh \kappa d\right) \cdot \mathrm{e}^{\mathrm{i} d A_{(j, h)}} \lambda_{(j, h)^{0} j^{\prime}}^{d}\left(k^{2}\right) \\
+\kappa \sum_{h \in N_{j}}\left(\mathrm{e}^{\mathrm{i} d A_{(j, h)}} \lambda_{(j, h)^{d} j^{\prime}}^{d}\left(k^{2}\right)\right)=\left(v_{j}-\mathrm{i} \sum_{h \in N_{j}} A_{(j, h)}\right) \lambda_{j j^{\prime}}^{d}\left(k^{2}\right), \\
\delta_{j l^{\prime}} \frac{\mathrm{e}^{\mathrm{i} d A_{\left(j, h^{\prime}\right)}} \sinh \kappa d}{\sin }-\kappa \lambda_{j\left(l, h^{\prime}\right)^{0}}^{d}\left(k^{2}\right)-\sum_{h \in N_{j}}\left(\kappa \cosh \kappa d+\mathrm{i} A_{(j, h)} \sinh \kappa d\right) \cdot \mathrm{e}^{\mathrm{i} d A_{(j, h)} \lambda_{(j, h)^{0}\left(l, h^{\prime}\right)^{0}}^{d}\left(k^{2}\right)} \\
+\kappa \sum_{h \in N_{j}}\left(\mathrm{e}^{\mathrm{i} d A_{(j, h)}} \lambda_{(j, h)^{d}\left(l, h^{\prime}\right)^{0}}^{d}\left(k^{2}\right)\right)=\left(v_{j}-\mathrm{i} \sum_{h \in N_{j}} A_{(j, h)}\right) \lambda_{j\left(l, h^{\prime}\right)^{0}}^{d}\left(k^{2}\right),
\end{array}
$$




$$
\begin{aligned}
-\kappa \lambda_{j\left(l, h^{\prime}\right)^{d}}^{d}\left(k^{2}\right)-\sum_{h \in N_{j}}(\kappa & \left.\cosh \kappa d+\mathrm{i} A_{(j, h)} \sinh \kappa d\right) \cdot \mathrm{e}^{\mathrm{i} d A_{(j, h)}} \lambda_{(j, h)^{0}\left(l, h^{\prime}\right)^{d}}^{d}\left(k^{2}\right) \\
& +\kappa \sum_{h \in N_{j}}\left(\mathrm{e}^{\mathrm{i} d A_{(j, h)}} \lambda_{(j, h)^{d}\left(l, h^{\prime}\right)^{d}}^{d}\left(k^{2}\right)\right)=\left(v_{j}-\mathrm{i} \sum_{h \in N_{j}} A_{(j, h)}\right) \lambda_{j\left(l, h^{\prime}\right)^{d}}^{d}\left(k^{2}\right) .
\end{aligned}
$$

We use the equalities (70) and (71) to eliminate all terms of the type $\lambda_{(j, h)^{0} J}^{d}\left(k^{2}\right)$ and $\lambda_{(j, h)^{d} J}^{d}\left(k^{2}\right)$, $J \in \hat{\mathcal{I}}$. In this way we obtain three independent systems of equations for $\lambda_{j j^{\prime}}^{d}\left(k^{2}\right)\left(j, j^{\prime} \in \hat{n}\right)$, $\lambda_{j\left(l^{\prime}, h^{\prime}\right)^{0}}^{d}\left(k^{2}\right)\left(j, l^{\prime} \in \hat{n}, h^{\prime} \in N_{l^{\prime}}\right)$ and $\lambda_{j\left(l^{\prime}, h^{\prime}\right)^{d}}^{d}\left(k^{2}\right)\left(j, l^{\prime} \in \hat{n}, h^{\prime} \in N_{l^{\prime}}\right)$ :

$$
\delta_{j j^{\prime}}-\kappa \lambda_{j j^{\prime}}^{d}\left(k^{2}\right)-\kappa \# N_{j} \frac{\cosh \kappa d}{\sinh \kappa d} \lambda_{j j^{\prime}}^{d}\left(k^{2}\right)+\sum_{h \in N_{j}} \frac{\kappa^{2}}{\sinh \kappa d} \cdot \frac{\lambda_{j j^{\prime}}^{d}\left(k^{2}\right)+\mathrm{e}^{2 \mathrm{i} d A_{(j, h)}} \lambda_{h j^{\prime}}^{d}\left(k^{2}\right)}{2 \kappa \cosh \kappa d+w_{\{j, h\}} \sinh \kappa d}=v_{j} \lambda_{j j^{\prime}}^{d}\left(k^{2}\right),
$$

$$
\begin{aligned}
\delta_{j l^{\prime}} \frac{\mathrm{e}^{\mathrm{i} d A_{\left(l^{\prime}, h^{\prime}\right)}}}{\sinh \kappa d}-\kappa \lambda_{j\left(l, h^{\prime}\right)^{0}}^{d}\left(k^{2}\right)-\kappa \# N_{j} \frac{\cosh \kappa d}{\sinh \kappa d} \lambda_{j\left(l, h^{\prime}\right)^{0}}^{d}\left(k^{2}\right) \\
+\sum_{h \in N_{j}} \frac{\kappa^{2}}{\sinh \kappa d} \cdot \frac{\lambda_{j\left(l, h^{\prime}\right)^{0}}^{d}\left(k^{2}\right)+\mathrm{e}^{2 \mathrm{i} d A_{(j, h)}} \lambda_{h\left(l, h^{\prime}\right)^{0}}^{d}\left(k^{2}\right)}{2 \kappa \cosh \kappa d+w_{\{j, h\}} \sinh \kappa d}=v_{j} \lambda_{j\left(l, h^{\prime}\right)^{0}}^{d}\left(k^{2}\right),
\end{aligned}
$$

$$
\begin{aligned}
-\kappa \lambda_{j\left(l, h^{\prime}\right) d}^{d}\left(k^{2}\right)-\kappa \# N_{j} & \frac{\cosh \kappa d}{\sinh \kappa d} \lambda_{j\left(l, h^{\prime}\right)^{d}}^{d}\left(k^{2}\right)+\sum_{h \in N_{j}} \frac{\kappa^{2}}{\sinh \kappa d} \cdot \frac{\lambda_{j\left(l, h^{\prime}\right)^{d}}^{d}\left(k^{2}\right)+\mathrm{e}^{2 \mathrm{i} d A_{(j, h)} \lambda_{h\left(l, h^{\prime}\right)^{d}}^{d}\left(k^{2}\right)}}{2 \kappa \cosh \kappa d+w_{\{j, h\}} \sinh \kappa d} \\
& +\frac{\kappa}{\sinh \kappa d} \cdot \sum_{h \in N_{j}} \frac{\mathrm{e}^{\mathrm{i} d A_{(j, h)}}}{2 \kappa \cosh \kappa d+w_{\{j, h\}} \sinh \kappa d} \delta_{j l^{\prime}} \delta_{h h^{\prime}}=v_{j} \lambda_{j\left(l, h^{\prime}\right)^{d}}^{d}\left(k^{2}\right) .
\end{aligned}
$$

Let us focus, e.g., on Eq. (74a), which can be rewritten in the form

$$
\begin{aligned}
\sum_{h=1}^{n}\left[\delta _ { j h } \left(\kappa+\kappa \# N_{j} \frac{\cosh \kappa d}{\sinh \kappa d}\right.\right. & \left.-\frac{\kappa}{\sinh \kappa d} \sum_{\tilde{h} \in N_{j}} \frac{\kappa}{2 \kappa \cosh \kappa d+w_{\{j, \tilde{h}} \sinh \kappa d}+v_{j}\right) \\
& \left.-\chi_{N_{j}}(h) \cdot \frac{\kappa}{\sinh \kappa d} \cdot \frac{\mathrm{e}^{2 \mathrm{i} d A_{(j, h)}}}{2 \kappa \cosh \kappa d+w_{\{j, h\}} \sinh \kappa d}\right] \lambda_{h j^{\prime}}^{d}\left(k^{2}\right)=\delta_{j j^{\prime}}
\end{aligned}
$$

for all $j, j^{\prime} \in \hat{n}$; the symbol $\chi_{N_{j}}(h)$ is equal to one if $h \in N_{j}$ holds and zero otherwise. As we will see within a short time, it is convenient to introduce a matrix $M_{d}$ the $(j, h)$-th element of which is defined by

$$
\begin{array}{r}
{\left[M_{d}\right]_{j h}=\delta_{j h}\left(\kappa+\kappa \# N_{j} \frac{\cosh \kappa d}{\sinh \kappa d}-\frac{\kappa}{\sinh \kappa d} \sum_{\tilde{h} \in N_{j}} \frac{\kappa}{2 \kappa \cosh \kappa d+w_{\{j, \tilde{h}\}} \sinh \kappa d}+v_{j}\right)} \\
-\chi_{N_{j}}(h) \cdot \frac{\kappa}{\sinh \kappa d} \cdot \frac{\mathrm{e}^{2 \mathrm{i} d A_{(j, h)}}}{2 \kappa \cosh \kappa d+w_{\{j, h\}} \sinh \kappa d} .
\end{array}
$$


We also rewrite the set $\hat{I}$ as a union,

$$
\hat{\mathcal{I}}=\hat{n} \cup \mathcal{J}^{0} \cup \mathcal{J}^{d},
$$

where $\mathcal{J}^{0}=\left\{(l, h)^{0} \mid l \in \hat{n}, h \in N_{l}\right\}$ and $\mathcal{J}^{d}=\left\{(l, h)^{d} \mid l \in \hat{n}, h \in N_{l}\right\}$, and define the symbols $\Lambda_{X Y}^{\mathrm{Ag}, d}\left(k^{2}\right)$ for $X, Y \in\left\{\hat{n}, \mathcal{J}^{0}, \mathcal{J}^{d}\right\}$ by the relation

$$
\Lambda_{X Y}^{\mathrm{Ag}, d}\left(k^{2}\right)=\left(\lambda_{J L}^{d}\left(k^{2}\right)\right)_{J \in X, L \in Y},
$$

e.g. $\Lambda_{\hat{n} \mathcal{J}^{0}}^{\mathrm{Ag}, d}\left(k^{2}\right)=\left(\lambda_{j\left(l^{\prime}, h^{\prime}\right)^{0}}^{d}\left(k^{2}\right)\right)_{j \in \hat{n},\left(l^{\prime}, h^{\prime}\right)^{0} \in \mathcal{T}^{0}}$. Obviously, the matrix $\Lambda^{\mathrm{Ag}, d}\left(k^{2}\right)$ has the block structure

$$
\Lambda^{\mathrm{Ag}, d}\left(k^{2}\right)=\left(\begin{array}{c|c|c}
\Lambda_{\hat{n} \hat{n}}^{\mathrm{Ag}, d}\left(k^{2}\right) & \Lambda_{\hat{n} \mathcal{J}^{0},}^{\mathrm{Ag}, d}\left(k^{2}\right) & \Lambda_{\hat{n} \mathcal{J}^{d}}^{\mathrm{Ag}, d}\left(k^{2}\right) \\
\hline \Lambda_{\mathcal{J}^{0} \hat{n}, d}^{\mathrm{Ag}}\left(k^{2}\right) & \Lambda_{\mathcal{J}^{0} d \mathcal{J}^{0}}^{\mathrm{Ag}}\left(k^{2}\right) & \Lambda_{\mathcal{J}^{0}, d}^{\mathrm{Ag} \mathcal{J}^{d}}\left(k^{2}\right) \\
\hline \Lambda_{\mathcal{J}^{d} \hat{n} \hat{n}}^{\mathrm{Ag}}\left(k^{2}\right) & \Lambda_{\mathcal{J}^{d} \mathcal{J}^{0}}^{\mathrm{Ag} d}\left(k^{2}\right) & \Lambda_{\mathcal{J}^{d} \mathcal{J}^{d}}^{\mathrm{Ag}}\left(k^{2}\right)
\end{array}\right) .
$$

We observe that the system of equations (75) is nothing but

$$
M_{d} \Lambda_{\hat{n} \hat{n}}^{\mathrm{Ag}, d}\left(k^{2}\right)=I,
$$

and therefore $\Lambda_{\hat{n} \hat{n}}^{\mathrm{Ag}, d}\left(k^{2}\right)=\left(M_{d}\right)^{-1}$, or in the components

$$
\lambda_{j j^{\prime}}^{d}\left(k^{2}\right)=\left[\left(M_{d}\right)^{-1}\right]_{j j^{\prime}} .
$$

The matrices $\Lambda_{\hat{n} \mathcal{T}^{0}}^{\mathrm{Ag}, d}\left(k^{2}\right)$ and $\Lambda_{\hat{n} \mathcal{J}^{d}}^{\mathrm{Ag}, d}\left(k^{2}\right)$ can be found in a similar way. We start from Eqs. (74b) and $(74 \mathrm{c})$ and arrive at

$$
\lambda_{j\left(l^{\prime}, h^{\prime}\right)^{0}}^{d}\left(k^{2}\right)=\frac{\mathrm{e}^{\mathrm{i} d A_{\left(l^{\prime}, h^{\prime}\right)}}}{\sinh \kappa d}\left[\left(M_{d}\right)^{-1}\right]_{j l^{\prime}}
$$

and

$$
\lambda_{j\left(l^{\prime}, h^{\prime}\right)^{d} d}^{d}\left(k^{2}\right)=\frac{\kappa}{\sinh \kappa d} \cdot \frac{\mathrm{e}^{\mathrm{i} d A_{\left(l^{\prime}, h^{\prime}\right)}}}{2 \kappa \cosh \kappa d+w_{\left\{l^{\prime}, h^{\prime}\right\}} \sinh \kappa d}\left[\left(M_{d}\right)^{-1}\right]_{j l^{\prime}} .
$$

To obtain expressions for $\Lambda_{\mathcal{J}^{0} X}^{\mathrm{Ag}, d}\left(k^{2}\right)$ and $\Lambda_{\mathcal{J}^{d} X}^{\mathrm{Ag}, d}\left(k^{2}\right)\left(X=\mathcal{J}^{0}, \mathcal{J}^{d}\right)$ we substitute (77a), (77b) and (77c) into Equations (70) and (71) which gives

$$
\begin{aligned}
& \lambda_{(l, h)^{0} j^{\prime}}^{d}\left(k^{2}\right)=\frac{\mathrm{e}^{-\mathrm{i} d A_{(, h)}}}{\sinh \kappa d} \cdot\left[\left(M_{d}\right)^{-1}\right]_{l j^{\prime}}, \\
& \lambda_{(l, h)^{0}\left(l^{\prime}, h^{\prime}\right)^{0}}^{d}\left(k^{2}\right)=\frac{\mathrm{e}^{-\mathrm{i} d A_{(l, h)}}}{\sinh \kappa d} \cdot \frac{\mathrm{e}^{\mathrm{i} d A_{\left(l^{\prime}, h^{\prime}\right)}}}{\sinh \kappa d}\left[\left(M_{d}\right)^{-1}\right]_{l^{\prime}}, \\
& \lambda_{(l, h)^{\circ}\left(l^{\prime}, h^{\prime}\right) d}^{d}\left(k^{2}\right)=\frac{\mathrm{e}^{-\mathrm{i} d A_{(l, h)}}}{\sinh ^{2} \kappa d} \cdot \frac{\kappa \cdot \mathrm{e}^{\mathrm{i} d A_{\left(l^{\prime}, h^{\prime}\right)}}}{2 \kappa \cosh \kappa d+w_{\left\{l^{\prime}, h^{\prime}\right\}} \sinh \kappa d}\left[\left(M_{d}\right)^{-1}\right]_{l l^{\prime}}, \\
& \lambda_{(l, h)^{d} j^{\prime}}^{d}\left(k^{2}\right)=\frac{\kappa}{2 \kappa \cosh \kappa d+w_{\{l, h\}} \sinh \kappa d} \cdot \frac{1}{\sinh \kappa d} \cdot\left(\mathrm{e}^{-\mathrm{i} d A_{(, h)}}\left[\left(M_{d}\right)^{-1}\right]_{l j^{\prime}}+\mathrm{e}^{-\mathrm{i} d A_{(h, h)}}\left[\left(M_{d}\right)^{-1}\right]_{h j^{\prime}}\right),
\end{aligned}
$$




$$
\begin{aligned}
& \lambda_{(l, h)^{d}\left(l^{\prime}, h^{\prime}\right)^{0}}^{d}\left(k^{2}\right)=\frac{\kappa}{2 \kappa \cosh \kappa d+w_{\{l, h\}} \sinh \kappa d} \cdot \frac{\mathrm{e}^{\mathrm{i} d A_{\left(l^{\prime}, h^{\prime}\right)}}}{\sinh ^{2} \kappa d} \cdot \\
& \cdot\left(\mathrm{e}^{-\mathrm{i} d A_{(l, h)}} \cdot\left[\left(M_{d}\right)^{-1}\right]_{l l^{\prime}}+\mathrm{e}^{-\mathrm{i} d A_{(h, l)}} \cdot\left[\left(M_{d}\right)^{-1}\right]_{h l^{\prime}}\right), \quad(77 \mathrm{~h}) \\
& \lambda_{(l, h)^{d}\left(l^{\prime}, h^{\prime}\right)^{d}}^{d}\left(k^{2}\right)=\frac{\kappa}{2 \kappa \cosh \kappa d+w_{\{l, h\}} \sinh \kappa d} \cdot \frac{1}{\sinh \kappa d} \cdot \\
& \cdot\left[\frac{\kappa}{\sinh \kappa d} \cdot \frac{\left.\mathrm{e}^{\mathrm{i} d A_{\left(l^{\prime}, h^{\prime}\right)}} \cdot \frac{1}{2 \kappa \cosh \kappa d+w_{\left\{l^{\prime}, h^{\prime}\right\}} \sinh \kappa d}\left(\mathrm{e}^{\left.-\mathrm{i} d A_{(l, h)}\right)} \cdot\left[\left(M_{d}\right)^{-1}\right]_{l l^{\prime}}+\mathrm{e}^{\left.-\mathrm{i} d A_{(h, l)}\right)} \cdot\left[\left(M_{d}\right)^{-1}\right]_{h l^{\prime}}\right)+\frac{1}{\kappa} \delta_{(l, h)\left(l^{\prime}, h^{\prime}\right)}\right] .}{(77 \mathrm{i})}\right.
\end{aligned}
$$

Once we compute the elements of $\left(M_{d}\right)^{-1}$ explicitly, we will have fully explicit formulae for $\Lambda^{\mathrm{Ag}, d}\left(k^{2}\right)$ we need. We start from the matrix $M_{d}$ itself. We take the formula (76), substitute there the expressions for $v_{j}(d), w_{\{j, k\}}(d)$ and $A_{(j, k)}(d)$ that have been obtained heuristically in the previous section and apply Taylor expansions to appropriate orders. A slightly laborious calculation leads to the formulae written below; note that the structure of the expression for the $(j, h)$-th element of the matrix $M_{d}$ depends on whether $j, h$ belong to $\hat{m}$ or to $\hat{n} \backslash \hat{m}$ :

$$
\begin{gathered}
{\left[M_{d}\right]_{j h}=\delta_{j h}\left(\kappa+S_{j j}+\frac{1}{d} \sum_{l=m+1}^{n} T_{j l} \overline{T_{l j}}\right)+S_{j h}+\frac{1}{d} \sum_{l=m+1}^{n} T_{j l} \overline{T_{h l}}+O(d) \quad \text { for } j, h \in \hat{m},} \\
{\left[M_{d}\right]_{j h}=-\frac{1}{d} T_{j h}+O(d) \quad \text { for } \quad j \in \hat{m}, h \geq m+1} \\
{\left[M_{d}\right]_{j h}=-\frac{1}{d} \overline{T_{h j}}+O(d) \quad \text { for } \quad j \geq m+1, h \in \hat{m}} \\
{\left[M_{d}\right]_{j h}=\delta_{j h}\left(\kappa+\frac{1}{d}\right)+O(d) \text { for } j, h \geq m+1 .}
\end{gathered}
$$

The matrix $M_{d}$ has thus the form

$$
M_{d}=\left(\begin{array}{c|c}
S+\kappa I^{(m)}+\frac{1}{d} T T^{*} & -\frac{1}{d} T \\
\hline-\frac{1}{d} T^{*} & \left(\kappa+\frac{1}{d}\right) I^{(n-m)}
\end{array}\right)+O(d),
$$

where $O(d)$ on the eight-hand side represents a matrix $n \times n$ the all entries of which are of order of $O(d)$ as $d \rightarrow 0$.

Our aim is to find the inverse of $M_{d}$. For this purpose, we denote the first term on the righthand side, the principal one, as $M_{d, P}$ and notice that if $M_{d, P}$ is regular, then

$$
\begin{aligned}
{\left[M_{d}\right]^{-1}=\left(M_{d, P}+O(d)\right)^{-1}=\left[M_{d, P}\left(I+\left[M_{d, P}\right]^{-1} O(d)\right)\right]^{-1}=} \\
=\left[I-\left[M_{d, P}\right]^{-1} O(d)\right]\left[M_{d, P}\right]^{-1}=\left[M_{d, P}\right]^{-1}-\left[M_{d, P}\right]^{-1} O(d)\left[M_{d, P}\right]^{-1}
\end{aligned}
$$

Moreover, if $\left[M_{d, P}\right]^{-1}=O(1)$ it obviously holds $\left[M_{d}\right]^{-1}=\left[M_{d, P}\right]^{-1}+O(d)$; in other words, under certain assumptions it suffices to find the inverse of $M_{d, P}$. 
Since the matrix $M_{d, P}$ has a block structure, one can find $M_{d, P}$ in the same block structure. This in other words means that we are looking for a matrix $\left(\begin{array}{l|l}N_{1} & N_{2} \\ \hline N_{3} & N_{4}\end{array}\right)$ such that the relation

$$
\left(\begin{array}{c|c}
N_{1} & N_{2} \\
\hline N_{3} & N_{4}
\end{array}\right) \cdot\left(\begin{array}{c|c}
S+\kappa I^{(m)}+\frac{1}{d} T T^{*} & -\frac{1}{d} T \\
\hline-\frac{1}{d} T^{*} & \left(\kappa+\frac{1}{d}\right) I^{(n-m)}
\end{array}\right)=\left(\begin{array}{c|c}
I^{(m)} & 0 \\
\hline 0 & I^{(n-m)}
\end{array}\right)
$$

holds true. It turns out that

$$
\begin{aligned}
& {\left[M_{d, P}\right]^{-1}=} \\
& \left.\qquad \begin{array}{cc}
\left(S+\kappa I^{(m)}+\frac{\kappa}{1+\kappa d} T T^{*}\right)^{-1} & \frac{1}{1+\kappa d}\left(S+\kappa I^{(m)}+\frac{\kappa}{1+\kappa d} T T^{*}\right)^{-1} T \\
\frac{1}{1+\kappa d} T^{*}\left(S+\kappa I^{(m)}+\frac{\kappa}{1+\kappa d} T T^{*}\right)^{-1} & \frac{1}{(1+\kappa d)^{2}} T^{*}\left(S+\kappa I^{(m)}+\frac{\kappa}{1+\kappa d} T T^{*}\right)^{-1} T+\frac{d}{1+\kappa d} I^{(n-m)}
\end{array}\right)
\end{aligned}
$$

provided the matrix $S+\kappa I^{(m)}+\frac{\kappa}{1+\kappa d} T T^{*}$ is regular. Since $\frac{\kappa}{1+\kappa d}=\kappa+O(d)$, we may proceed in the same way as in (79), and we obtain

$$
\left(S+\kappa I^{(m)}+\frac{\kappa}{1+\kappa d} T T^{*}\right)^{-1}=\left(S+\kappa I^{(m)}+\kappa T T^{*}+O(d)\right)^{-1}=\left(S+\kappa I^{(m)}+\kappa T T^{*}\right)^{-1}+O(d),
$$

if the matrix $S+\kappa I^{(m)}+\kappa T T^{*}$ is regular. However, the regularity of this matrix has been discussed and proven for an appropriate $\kappa$ at the end of the part devoted to the approximated system, see Eq. (56) and the paragraph following it. It follows that $M_{d, P}$ is regular as well, i.e. the condition on the regularity of $M_{d, P}$ in (79) is satisfied. Hence

$$
\left[M_{d, P}\right]^{-1}=\left(\begin{array}{cc}
\left(S+\kappa I^{(m)}+\kappa T T^{*}\right)^{-1} & \left(S+\kappa I^{(m)}+\kappa T T^{*}\right)^{-1} T \\
T^{*}\left(S+\kappa I^{(m)}+\kappa T T^{*}\right)^{-1} & T^{*}\left(S+\kappa I^{(m)}+\kappa T T^{*}\right)^{-1} T
\end{array}\right)+O(d),
$$

and together with (79) we have

$$
\left[M_{d}\right]^{-1}=\left(\begin{array}{cc}
\left(S+\kappa I^{(m)}+\kappa T T^{*}\right)^{-1} & \left(S+\kappa I^{(m)}+\kappa T T^{*}\right)^{-1} T \\
T^{*}\left(S+\kappa I^{(m)}+\kappa T T^{*}\right)^{-1} & T^{*}\left(S+\kappa I^{(m)}+\kappa T T^{*}\right)^{-1} T
\end{array}\right)+O(d) .
$$

It is important to notice that

$$
\left[M_{d}\right]^{-1}=O(1) \text { for } d \rightarrow 0_{+} .
$$

Combining the above result with Eq. (77a), we can conclude that

$$
\Lambda_{\hat{n} \hat{n}}^{\mathrm{Ag}, d}\left(k^{2}\right)=\left(\begin{array}{cc}
\left(S+\kappa I^{(m)}+\kappa T T^{*}\right)^{-1} & \left(S+\kappa I^{(m)}+\kappa T T^{*}\right)^{-1} T \\
T^{*}\left(S+\kappa I^{(m)}+\kappa T T^{*}\right)^{-1} & T^{*}\left(S+\kappa I^{(m)}+\kappa T T^{*}\right)^{-1} T
\end{array}\right)+O(d),
$$

hence

$$
\Lambda_{\hat{n} \hat{n}}^{\mathrm{Ag}, d}\left(k^{2}\right)=\Lambda^{\mathrm{Ad}}\left(k^{2}\right)+O(d) .
$$

Having the coefficient matrix we can determine the resolvent kernel. First we introduce symbol $\mathcal{J}=\left\{(l, h) \mid l \in \hat{n}, h \in N_{l}\right\}$ (i.e. $\mathcal{I}=\hat{n} \cup \mathcal{J}$ ), then we employ a notation similar to the case of the matrix $\Lambda^{\mathrm{Ag}, d}\left(k^{2}\right)$ and its submatrices. We introduce symbols $\mathcal{G}_{X Y, k}^{\mathrm{Ag}, d}$ for any pair $X, Y \in$ $\{\hat{n}, \mathcal{J}\}$ to denote the blocks $\mathcal{G}_{k, X Y}^{\mathrm{Ag}, d}=\left(\mathcal{G}_{k, J L}^{\mathrm{Ag}, d}\right)_{J \in X, L \in Y}$; then the integral kernel $\mathcal{G}_{k}^{\mathrm{Ag}, d}$ of $R_{d}^{\mathrm{Ag}}\left(k^{2}\right)$ has the structure

$$
\mathcal{G}_{k}^{\mathrm{Ag}, d}(x, y)=\left(\begin{array}{c|c}
\mathcal{G}_{k, \hat{n} \hat{n}}^{\mathrm{Ag}, d}(x, y) & \mathcal{G}_{k, \hat{n} \mathcal{J}}^{\mathrm{Ag}, d}(x, y) \\
\hline \mathcal{G}_{k, \mathcal{J} \hat{n}}^{\mathrm{Ag}, d}(x, y) & \mathcal{G}_{k, \mathcal{J} \mathcal{J}}^{\mathrm{Ag}, d}(x, y)
\end{array}\right)
$$


for $x, y \in G_{d}$. Using (58) we can write the difference in question as

$$
\mathcal{G}_{\mathrm{i} \kappa}^{\mathrm{Ag}, d}-\mathcal{G}_{\mathrm{i} \kappa}^{\mathrm{Ad}, d}=\left(\begin{array}{c|c}
\mathcal{G}_{\mathrm{i} \kappa, \hat{n} \hat{n}}^{\mathrm{Ag}, d}-\mathcal{G}_{\mathrm{i} \kappa}^{\mathrm{Ad}} & \mathcal{G}_{\mathrm{i} \kappa}^{\mathrm{Ag}, \hat{n} \mathcal{J}} \\
\hline \mathcal{G}_{\mathrm{i} \kappa, \hat{n} \mathcal{J}}^{\mathrm{g}} & \mathcal{G}_{\mathrm{i} \kappa, \mathcal{J} \mathcal{J}}^{\mathrm{Ag}, d}
\end{array}\right) .
$$

\section{Comparison of the resolvents}

To make use of the above results we compute first explicit expressions for all the entries of $\mathcal{G}_{k}^{\mathrm{Ag}, d}(x, y)$, up to the error term in the lambda coefficients indicated in (81). They may be derived from Eqs (62) together with (61):

$$
\begin{aligned}
& \mathcal{G}_{\mathrm{i} \kappa, j j^{\prime}}^{\mathrm{Ag}, d}\left(x_{j}, y_{j^{\prime}}\right)=\delta_{j j^{\prime}} \frac{\sinh \kappa x_{<} \mathrm{e}^{-\kappa x_{>}}}{\kappa}+\lambda_{j j^{\prime}}^{d}\left(k^{2}\right) \mathrm{e}^{-\kappa x_{j}} \mathrm{e}^{-\kappa y_{j^{\prime}}} \quad \text { for } \quad j, j^{\prime} \in \hat{n},
\end{aligned}
$$

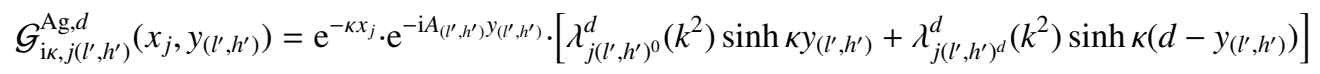

$$
\begin{aligned}
& \text { for } \quad j \in \hat{n},\left(l^{\prime}, h^{\prime}\right) \in \mathcal{J}, \quad(84 \mathrm{~b}) \\
& \mathcal{G}_{\mathrm{i} \kappa,(l, h) j^{\prime}}^{\mathrm{Ag}, d}\left(x_{(l, h)}, y_{j^{\prime}}\right)=\mathrm{e}^{\mathrm{i} A_{(l, h)} x_{(l, h)}} \cdot\left[\lambda_{(l, h)^{0} j^{\prime}}^{d}\left(k^{2}\right) \sinh \kappa x_{(l, h)}+\lambda_{(l, h)^{d} j^{\prime}}^{d}\left(k^{2}\right) \sinh \kappa\left(d-x_{(l, h)}\right)\right] \cdot \mathrm{e}^{-\kappa y_{j^{\prime}}} \\
& \text { for }(l, h) \in \mathcal{J}, j^{\prime} \in \hat{n}, \\
& \mathcal{G}_{\mathrm{i} \kappa,(l, h)\left(l^{\prime}, h^{\prime}\right)}^{\mathrm{Ag}, d}\left(x_{(l, h)}, y_{\left(l^{\prime}, h^{\prime}\right)}\right)=\delta_{(l, h)\left(l^{\prime}, h^{\prime}\right)} \mathrm{e}^{\mathrm{i} A_{(l, h)} x_{(l, h)}} \frac{\sinh \kappa x_{<} \sinh \kappa\left(d-x_{>}\right)}{\kappa \sinh \kappa d} \mathrm{e}^{-\mathrm{i} A_{\left(l^{\prime}, h^{\prime}\right.} y_{\left(l^{\prime}, h^{\prime}\right)}} \\
& +\mathrm{e}^{\mathrm{i} A_{(l, h)} x_{(l, h)}} \cdot \sinh \kappa x_{(l, h)} \cdot \mathrm{e}^{-\mathrm{i} A_{\left(l^{\prime}, h^{\prime}\right)} y_{\left(l^{\prime}, h^{\prime}\right)}} \cdot\left[\lambda_{(l, h)^{0}\left(l^{\prime} h^{\prime}\right)^{0}}^{d} \sinh \kappa y_{\left(l^{\prime}, h^{\prime}\right)}+\lambda_{(l, h)^{0}\left(l^{\prime} h^{\prime}\right)^{d}}^{d} \sinh \kappa\left(d-y_{\left(l^{\prime}, h^{\prime}\right)}\right)\right] \\
& +\mathrm{e}^{\mathrm{i} A_{(l, h)} x_{(l, h)}} \cdot \sinh \kappa\left(d-x_{(l, h)}\right) \cdot \mathrm{e}^{-\mathrm{i} A_{\left(l^{\prime}, h^{\prime}\right)} y_{\left(l^{\prime}, h^{\prime}\right)}} \cdot\left[\lambda_{(l, h)^{d}\left(l^{\prime} h^{\prime}\right)^{0}}^{d} \sinh \kappa y_{\left(l^{\prime}, h^{\prime}\right)}+\lambda_{(l, h)^{d}\left(l^{\prime} h^{\prime}\right)^{d}}^{d} \sinh \kappa\left(d-y_{\left(l^{\prime}, h^{\prime}\right)}\right)\right] \\
& \text { for } \quad(l, h),\left(l^{\prime}, h^{\prime}\right) \in \mathcal{J} . \quad(84 \mathrm{~d})
\end{aligned}
$$

Now we are able to compare the entries of $\mathcal{G}_{k}^{\mathrm{Ag}, d}(x, y)$ given by (82) and $\mathcal{G}_{k}^{A d, d}(x, y)$ as specified in (58)). We begin with the upper left submatrix $n \times n$ of (83). From the expressions for $\mathcal{G}_{\mathrm{i} k}^{\mathrm{Ad}}$ and $\mathcal{G}_{\mathrm{i} \kappa, \hat{n} \hat{n}}^{\mathrm{Ag},}$, cf. (57) and (84a), we have

$$
\begin{aligned}
& {\left[\mathcal{G}_{\mathrm{i} \kappa, \hat{n} \hat{n}}^{\mathrm{Ag}, d}-\mathcal{G}_{\mathrm{i} \kappa}^{\mathrm{Ad}}\right]_{j j^{\prime}}\left(x_{j}, y_{j^{\prime}}\right)} \\
& =\delta_{j j^{\prime}} \frac{\sinh \kappa x_{<} \mathrm{e}^{-\kappa x_{>}}}{\kappa}+\lambda_{j j^{\prime}}^{d}\left(k^{2}\right) \mathrm{e}^{-\kappa x_{j}} \mathrm{e}^{-\kappa y_{j^{\prime}}}-\left[\delta_{j l} \frac{\sinh \kappa x_{<} \mathrm{e}^{-\kappa x_{>}}}{\kappa}+\lambda_{j j^{\prime}}\left(k^{2}\right) \mathrm{e}^{-\kappa x_{j}} \mathrm{e}^{-\kappa y_{j^{\prime}}}\right] \\
& =\left(\lambda_{j j^{\prime}}^{d}\left(k^{2}\right)-\lambda_{j j^{\prime}}\left(k^{2}\right)\right) \mathrm{e}^{-\kappa x_{j}} \mathrm{e}^{-\kappa y_{j^{\prime}}}=\left[\Lambda_{\hat{n} \hat{n}}^{\mathrm{Ag}, d}\left(k^{2}\right)-\Lambda^{\mathrm{Ad}}\left(k^{2}\right)\right]_{j j^{\prime}} \mathrm{e}^{-\kappa x_{j}} \mathrm{e}^{-\kappa y_{j^{\prime}}}=O(d) \mathrm{e}^{-\kappa x_{j}} \mathrm{e}^{-\kappa y_{j^{\prime}}},
\end{aligned}
$$

where the last equality holds by virtue of (81). Since such estimate is valid for all $j, j^{\prime} \in \hat{n}$, there is a constant $K_{1}$ independent of $j, j^{\prime}$ and $d$ such that

$$
\left|\mathcal{G}_{\mathrm{i} \kappa, j j^{\prime}}^{\mathrm{Ag}, d}\left(x_{j}, y_{j^{\prime}}\right)-\mathcal{G}_{\mathrm{i} \kappa, j j^{\prime}}^{\mathrm{Ad}}\left(x_{j}, y_{j^{\prime}}\right)\right|<K_{1} d \mathrm{e}^{-\kappa x_{j}} \mathrm{e}^{-\kappa y_{j^{\prime}}}
$$


holds for all $j, j^{\prime} \in \hat{n}, x_{j}, y_{j^{\prime}} \in \mathbb{R}^{+}$and any $d$ sufficiently small.

Then we proceed to the upper right submatrix of (83). To find a bound for the entries of $\mathcal{G}_{k, \hat{n} \mathcal{J}}^{\mathrm{Ag}, d}(x, y)$, cf. (84b), we substitute values of $\lambda_{j\left(l^{\prime}, h^{\prime}\right)^{0}}^{d}\left(k^{2}\right)$ and $\lambda_{j\left(l^{\prime}, h^{\prime}\right)^{d}}^{d}\left(k^{2}\right)$ that we have obtained in (77b) and (77c):

$$
\begin{aligned}
& \mathcal{G}_{\mathrm{i} \kappa, j\left(l^{\prime}, h^{\prime}\right)}^{\mathrm{Ag}, d}\left(x_{j}, y_{\left(l^{\prime}, h^{\prime}\right)}\right)= \\
& \mathrm{e}^{-\kappa x_{j}} \cdot \mathrm{e}^{-\mathrm{i} A_{\left(l^{\prime}, h^{\prime}\right)} y_{\left(l^{\prime}, h^{\prime}\right)}} \cdot \mathrm{e}^{\mathrm{i} d A_{\left(l^{\prime}, h^{\prime}\right)}} \cdot\left[\frac{\sinh \kappa y_{\left(l^{\prime}, h^{\prime}\right)}}{\sinh \kappa d}+\frac{\kappa}{\sinh \kappa d} \cdot \frac{\sinh \kappa\left(d-y_{\left(l^{\prime}, h^{\prime}\right)}\right)}{2 \kappa \cosh \kappa d+w_{\left\{l^{\prime}, h^{\prime}\right\}} \sinh \kappa d}\right] \cdot\left[\left(M_{d}\right)^{-1}\right]_{j l^{\prime}} .
\end{aligned}
$$

It holds $\left[\left(M_{d}\right)^{-1}\right]_{j l^{\prime}}=O(1)$ by virtue of (80) and obviously $\left|\mathrm{e}^{-\mathrm{i} A_{\left(l^{\prime}, h^{\prime}\right)} y_{\left(l^{\prime}, h^{\prime}\right)}}\right|=\left|\mathrm{e}^{\mathrm{i} d A_{\left(l^{\prime}, h^{\prime}\right)}}\right|=1$, thus it suffices to estimate the terms in the brackets. When $d$ is sufficiently small, it holds $\left|\frac{\sinh \kappa y_{\left(l^{\prime}, h^{\prime}\right)}}{\sinh \kappa d}\right|<1$, because $0<y_{\left(l^{\prime}, h^{\prime}\right)}<d$; similarly $\left|\frac{\sinh \kappa\left(d-y_{\left(l^{\prime}, h^{\prime}\right)}\right)}{\sinh \kappa d}\right|<1$. As for the denominator of the second term, we substitute for $w_{\left\{l^{\prime}, h^{\prime}\right\}}$ from (49) or (47), depending on whether both $l^{\prime}, h^{\prime}$ belong to $\hat{m}$ or not, and we easily obtain the estimate

$$
\frac{1}{2 \kappa \cosh \kappa d+w_{\left\{l^{\prime}, h^{\prime}\right\}} \sinh \kappa d}=O(1)
$$

Summing all this up, we get

$$
\mathcal{G}_{\mathrm{i} \kappa, j\left(l^{\prime}, h^{\prime}\right)}^{\mathrm{Ag}, d}\left(x_{j}, y_{\left(l^{\prime}, h^{\prime}\right)}\right)=\mathrm{e}^{-\kappa x_{j}}(O(1)+O(1))=\mathrm{e}^{-\kappa x_{j}} O(1)
$$

independently of $j,\left(l^{\prime}, h^{\prime}\right)$ and $x, y$, thus there is a constant $K_{2}$ independent of $d$ such that

$$
\left|\mathcal{G}_{\mathrm{i} \kappa, j\left(l^{\prime}, h^{\prime}\right)}^{\mathrm{Ag}, d}\left(x_{j}, y_{\left(l^{\prime}, h^{\prime}\right)}\right)\right|<K_{2} \mathrm{e}^{-\kappa x_{j}}
$$

for all $j \in \hat{n},\left(l^{\prime}, h^{\prime}\right) \in \mathcal{J}, x_{j} \in \mathbb{R}^{+}, y_{\left(l^{\prime}, h^{\prime}\right)} \in(0, d)$ and $d$ sufficiently small.

Similarly we proceed in the case of the left and right bottom submatrices of (83), i.e. when estimating the entries of $\mathcal{G}_{k, \mathcal{J} \hat{n}}^{\mathrm{Ag}, d}(x, y)$ and $\mathcal{G}_{k, \mathcal{J} \mathcal{J}}^{\mathrm{Ag}, d}(x, y)$. As for $\mathcal{G}_{k, \mathcal{J} \hat{n}}^{\mathrm{Ag}, d}(x, y)$, we substitute for $\lambda_{(l, h)^{0} j^{\prime}}\left(k^{2}\right)$ and $\lambda_{(l, h)^{d} j^{\prime}}^{d}\left(k^{2}\right)$ from $(77 \mathrm{~d})$ and $(77 \mathrm{~g})$ into $(84 \mathrm{c})$ and obtain

$$
\begin{aligned}
& \mathcal{G}_{\mathrm{i} \kappa,(l, h) j^{\prime}}^{\mathrm{Ag}, d}\left(x_{(l, h)}, y_{j^{\prime}}\right)= \mathrm{e}^{-\kappa y_{j^{\prime}}} \cdot \mathrm{e}^{\mathrm{i} A_{(l, h)} x_{(l, h)}} \cdot\left[\frac{\mathrm{e}^{-\mathrm{i} d A_{(l, h)}}}{\sinh \kappa d} \cdot\left[\left(M_{d}\right)^{-1}\right]_{l j^{\prime}} \sinh \kappa x_{(l, h)}\right. \\
&+\frac{\kappa}{2 \kappa \cosh \kappa d+w_{\{l, h\}} \sinh \kappa d} \cdot \frac{1}{\sinh \kappa d} \cdot \sinh \kappa\left(d-x_{(l, h)}\right) . \\
&\left.\cdot\left(\mathrm{e}^{-\mathrm{i} d A_{(l, h)}}\left[\left(M_{d}\right)^{-1}\right]_{l j^{\prime}}+\mathrm{e}^{-\mathrm{i} d A_{(h, l)}}\left[\left(M_{d}\right)^{-1}\right]_{h j^{\prime}}\right)\right] .
\end{aligned}
$$

Using analogous estimates as in the case of $\mathcal{G}_{\mathrm{i} \kappa, j\left(l^{\prime}, h^{\prime}\right)}^{\mathrm{Ag}, d}(x, y)$ above, we obtain

$$
\mathcal{G}_{\mathrm{i} \kappa,(l, h) j^{\prime}}^{\mathrm{Ag}, d}\left(x_{(l, h)}, y_{j^{\prime}}\right)=\mathrm{e}^{-\kappa y_{j^{\prime}}}(O(1)+O(1))=\mathrm{e}^{-\kappa y} y_{j^{\prime}} O(1),
$$

thus there is a constant $K_{3}$ independent of $d$ such that

$$
\left|\mathcal{G}_{\mathrm{i} \kappa,(l, h) j^{\prime}}^{\mathrm{Ag}, d}\left(x_{(l, h)}, y_{j^{\prime}}\right)\right|<K_{3} \mathrm{e}^{-\kappa y_{j^{\prime}}}
$$


for all $(l, h) \in \mathcal{J}, j^{\prime} \in \hat{n}, x_{(l, h)} \in(0, d), y \in \mathbb{R}^{+}$and $d$ sufficiently small.

Finally, we substitute from (77e), (77f), (77h) and (77i) for $\lambda_{(l, h)^{0}\left(l^{\prime} h^{\prime}\right)^{0}}^{d}\left(k^{2}\right), \lambda_{(l, h)^{0}\left(l^{\prime} h^{\prime}\right)^{d}}^{d}\left(k^{2}\right)$, $\lambda_{(l, h)^{d}\left(l^{\prime} h^{\prime}\right)^{0}}^{d}\left(k^{2}\right)$ and $\lambda_{(l, h)^{d}\left(l^{\prime} h^{\prime}\right)^{d}}^{d}\left(k^{2}\right)$, respectively, into Eq. (84d) and obtain

$$
\begin{aligned}
& \mathcal{G}_{\mathrm{i} \kappa,(l, h)\left(l^{\prime}, h^{\prime}\right)}^{\mathrm{Ag}, d}\left(x_{(l, h)}, y_{\left(l^{\prime}, h^{\prime}\right)}\right)=\delta_{(l, h)\left(l^{\prime}, h^{\prime}\right)} \mathrm{e}^{\mathrm{i} A_{(l, h)} x_{(l, h)}} \frac{\sinh \kappa x_{<} \sinh \kappa\left(d-x_{>}\right)}{\kappa \sinh \kappa d} \mathrm{e}^{-\mathrm{i} A_{\left(l^{\prime}, h^{\prime}\right)} y_{\left(l^{\prime}, h^{\prime}\right)}} \\
& +\mathrm{e}^{\mathrm{i} A_{(l, h)} x_{(l, h)}} \cdot \mathrm{e}^{-\mathrm{i} A_{\left(l^{\prime}, h^{\prime}\right)} y_{\left(l^{\prime}, h^{\prime}\right)}} \cdot \mathrm{e}^{-\mathrm{i} d A_{(l, h)}} \cdot \mathrm{e}^{\mathrm{i} d A_{\left(l^{\prime}, h^{\prime}\right)}} \cdot \frac{\sinh \kappa x_{(l, h)}}{\sinh \kappa d} \cdot \\
& \cdot\left[\frac{\sinh \kappa y_{\left(l^{\prime}, h^{\prime}\right)}}{\sinh \kappa d}+\frac{\kappa}{\sinh \kappa d} \cdot \frac{\sinh \kappa\left(d-y_{\left(l^{\prime}, h^{\prime}\right)}\right)}{2 \kappa \cosh \kappa d+w_{\left\{l^{\prime}, h^{\prime}\right\}} \sinh \kappa d}\right] \cdot\left[\left(M_{d}\right)^{-1}\right]_{l l^{\prime}} \\
& +\mathrm{e}^{\mathrm{i} A_{(l, h)} x_{(l, h)}} \cdot \sinh \kappa\left(d-x_{(l, h)}\right) \cdot \mathrm{e}^{-\mathrm{i} A_{\left(l^{\prime}, h^{\prime}\right)} y_{\left(l^{\prime}, h^{\prime}\right)}} \cdot \frac{\kappa}{2 \kappa \cosh \kappa d+w_{\{l, h\}} \sinh \kappa d} \cdot \frac{1}{\sinh \kappa d} . \\
& \cdot\left[\mathrm{e}^{\mathrm{i} d A_{\left(l^{\prime}, h^{\prime}\right)}} \cdot \frac{\sinh \kappa y_{\left(l^{\prime}, h^{\prime}\right)}}{\sinh \kappa d}\left(\mathrm{e}^{-\mathrm{i} d A_{(l, h)}} \cdot\left[\left(M_{d}\right)^{-1}\right]_{l l^{\prime}}+\mathrm{e}^{-\mathrm{i} d A_{(h, l)}} \cdot\left[\left(M_{d}\right)^{-1}\right]_{h l^{\prime}}\right)\right. \\
& +\mathrm{e}^{\mathrm{i} d A_{\left(l^{\prime}, h^{\prime}\right)}} \cdot \frac{\kappa}{\sinh \kappa d} \cdot \frac{\sinh \kappa\left(d-y_{\left(l^{\prime}, h^{\prime}\right)}\right)}{2 \kappa \cosh \kappa d+w_{\left\{l^{\prime}, h^{\prime}\right\}} \sinh \kappa d} \text {. } \\
& \left.\cdot\left(\mathrm{e}^{-\mathrm{i} d A_{(l, h)}} \cdot\left[\left(M_{d}\right)^{-1}\right]_{l l^{\prime}}+\mathrm{e}^{-\mathrm{i} d A_{(h, l)}} \cdot\left[\left(M_{d}\right)^{-1}\right]_{h l^{\prime}}\right)+\frac{1}{\kappa} \delta_{(l, h)\left(l^{\prime}, h^{\prime}\right)}\right] \cdot
\end{aligned}
$$

It obviously holds

$$
\mathcal{G}_{\mathrm{i} K,(l, h)\left(l^{\prime}, h^{\prime}\right)}^{\mathrm{Ag},}\left(x_{(l, h)}, y_{\left(l^{\prime}, h^{\prime}\right)}\right)==O(d)+O(1) \cdot[O(1)+O(1)]+O(1) \cdot[O(1)+O(1)]=O(1),
$$

thus there is a constant $K_{4}$ independent of $d$ such that

$$
\left|\mathcal{G}_{\mathrm{i} \kappa,(l, h)\left(l^{\prime}, h^{\prime}\right)}^{\mathrm{Ag}, d}\left(x_{(l, h)}, y_{\left(l^{\prime}, h^{\prime}\right)}\right)\right|<K_{4}
$$

for all $(l, h),\left(l^{\prime}, h^{\prime}\right) \in \mathcal{J}, x_{(l, h)}, y_{\left(l^{\prime}, h^{\prime}\right)} \in(0, d)$ and any $d$ sufficiently small.

With the help of (86), (87), (88) and (90), we may now estimate all the entries of (83), which will allows us to assess the Hilbert-Schmidt norm of the resolvent difference for the operators $H_{d}^{\mathrm{Ad}}$ and $H_{d}^{\mathrm{Ag}}$. This norm can be written explicitly as follows,

$$
\begin{aligned}
\left\|R_{d}^{\mathrm{Ag}}\left(k^{2}\right)-R_{d}^{\mathrm{Ad}}\left(k^{2}\right)\right\|_{2}^{2} & =\sum_{j, j^{\prime}=1}^{n} \int_{0}^{+\infty} \int_{0}^{+\infty}\left|\mathcal{G}_{\mathrm{i} \kappa, j j^{\prime}}^{\mathrm{Ag}, d}\left(x_{j}, y_{j^{\prime}}\right)-\mathcal{G}_{\mathrm{i} \kappa, j j^{\prime}}^{\mathrm{Ad}}\left(x_{j}, y_{j^{\prime}}\right)\right|^{2} \mathrm{~d} x_{j} \mathrm{~d} y_{j^{\prime}} \\
& +\sum_{j=1}^{n} \sum_{\left(l^{\prime}, h^{\prime}\right) \in \mathcal{I}} \int_{0}^{+\infty} \int_{0}^{d}\left|\mathcal{G}_{\mathrm{i} \kappa, j\left(l^{\prime}, h^{\prime}\right)}^{\mathrm{Ag}, d}\left(x_{j}, y_{\left(l^{\prime}, h^{\prime}\right)}\right)\right|^{2} \mathrm{~d} x_{j} \mathrm{~d} y_{\left(l^{\prime}, h^{\prime}\right)} \\
& +\sum_{(l, h) \in \mathcal{I}} \sum_{j^{\prime}=1}^{n} \int_{0}^{d} \int_{0}^{+\infty}\left|\mathcal{G}_{\mathrm{i} \kappa,(l, h) j^{\prime}}^{\mathrm{Ag}, d}\left(x_{(l, h)}, y_{j^{\prime}}\right)\right|^{2} \mathrm{~d} x_{(l, h)} \mathrm{d} y_{j^{\prime}} \\
& +\sum_{(l, h) \in \mathcal{I}} \sum_{\left(l^{\prime}, h^{\prime}\right) \in \mathcal{I}} \int_{0}^{d} \int_{0}^{d}\left|\mathcal{G}_{\mathrm{ig},(l, h)\left(l^{\prime} h^{\prime}\right)}^{\mathrm{Ag}, d}\left(x_{(l, h)}, y_{\left(l, h^{\prime}\right)}\right)\right|^{2} \mathrm{~d} x_{(l, h)} \mathrm{d} y_{\left(l^{\prime}, h^{\prime}\right)} .
\end{aligned}
$$


Now we employ the estimates derived above obtaining

$$
\begin{aligned}
& \left\|R_{d}^{\mathrm{Ag}}\left(k^{2}\right)-R_{d}^{\mathrm{Ad}}\left(k^{2}\right)\right\|_{2}^{2} \leq \\
& \sum_{j, j^{\prime}=1}^{n} \int_{0}^{+\infty} \int_{0}^{+\infty}\left|K_{1} d \mathrm{e}^{-\kappa x_{j}} \mathrm{e}^{-\kappa y_{j^{\prime}}}\right|^{2} \mathrm{~d} x_{j} \mathrm{~d} y_{j^{\prime}}+\sum_{j=1}^{n} \sum_{\left(l^{\prime}, h^{\prime}\right) \in \mathcal{I}} \int_{0}^{+\infty} \int_{0}^{d}\left|K_{2} \mathrm{e}^{-\kappa x_{j}}\right|^{2} \mathrm{~d} x_{j} \mathrm{~d} y_{\left(l^{\prime}, h^{\prime}\right)} \\
& +\sum_{(l, h) \in \mathcal{I}} \sum_{j^{\prime}=1}^{n} \int_{0}^{d} \int_{0}^{+\infty}\left|K_{3} \mathrm{e}^{-\kappa y_{j^{\prime}}}\right|^{2} \mathrm{~d} x_{(l, h)} \mathrm{d} y_{j^{\prime}}+\sum_{(l, h) \in \mathcal{I}} \sum_{\left(l^{\prime}, h^{\prime}\right) \in \mathcal{I}} \int_{0}^{d} \int_{0}^{d}\left|K_{4}\right|^{2} \mathrm{~d} x_{(l, h)} \mathrm{d} y_{\left(l^{\prime}, h^{\prime}\right)} \\
& \leq \sum_{j, j^{\prime}=1}^{n} K_{1}^{2} d^{2} \int_{0}^{+\infty} e^{-2(\operatorname{Re} \kappa) x_{j}} \mathrm{~d} x_{j} \int_{0}^{+\infty} e^{-2(\operatorname{Re} \kappa) y_{j^{\prime}}} \mathrm{d} y_{j^{\prime}} \\
& +\sum_{j=1}^{n} \sum_{\left(l^{\prime}, h^{\prime}\right) \in \mathcal{I}} K_{2}^{2} \int_{0}^{+\infty} e^{-2(\operatorname{Re} \kappa) x_{j}} \mathrm{~d} x_{j} \cdot \int_{0}^{d} 1 \mathrm{~d} y_{\left(l^{\prime}, h^{\prime}\right)} \\
& +\sum_{(l, h) \in \mathcal{I}} \sum_{j^{\prime}=1}^{n} K_{3}^{2} \int_{0}^{d} 1 \mathrm{~d} x_{(l, h)} \cdot \int_{0}^{+\infty} e^{-2(\operatorname{Re} \kappa) y_{j^{\prime}}} \mathrm{d} y_{j^{\prime}} \\
& +\sum_{(l, h) \in \mathcal{I}} \sum_{\left(l^{\prime}, h^{\prime}\right) \in \mathcal{I}} K_{4}^{2} \int_{0}^{d} 1 \mathrm{~d} x_{(l, h)} \cdot \int_{0}^{d} 1 \mathrm{~d} y_{\left(l^{\prime}, h^{\prime}\right)} \\
& =\sum_{j, j^{\prime}=1}^{n} K_{1}^{2} d^{2} \frac{1}{(2 \operatorname{Re} \kappa)^{2}}+\sum_{j=1}^{n} \sum_{\left(l^{\prime}, h^{\prime}\right) \in \mathcal{I}} K_{2}^{2} \frac{1}{2 \operatorname{Re} \kappa} \cdot d+\sum_{(l, h) \in \mathcal{I}} \sum_{j^{\prime}=1}^{n} K_{3}^{2} d \cdot \frac{1}{2 \operatorname{Re} \kappa}+\sum_{(l, h) \in \mathcal{I}} \sum_{\left(l^{\prime}, h^{\prime}\right) \in \mathcal{I}} K_{4}^{2} d^{2} \\
& =O(d) \text {. }
\end{aligned}
$$

Hence

$$
\left\|R_{d}^{\mathrm{Ag}}\left(k^{2}\right)-R_{d}^{\mathrm{Ad}}\left(k^{2}\right)\right\|_{2}=O(\sqrt{d}) \quad \text { for } d \rightarrow 0_{+},
$$

and consequently, the Hilbert-Schmidt norm of the difference $R_{d}^{\mathrm{Ag}}\left(k^{2}\right)-R_{d}^{\mathrm{Ad}}\left(k^{2}\right)$ tends to zero as $d \rightarrow 0_{+}$with the explicit convergence rate. Since the HS norm dominates the operator one, it follows immediately

$$
\lim _{d \rightarrow 0_{+}}\left\|R_{d}^{\mathrm{Ag}}\left(k^{2}\right)-R_{d}^{\mathrm{Ad}}\left(k^{2}\right)\right\|=0
$$

therefore the resolvent difference tends to zero in $L^{2}\left(G_{d}\right)$ as $d \rightarrow 0_{+}$, which we set out to prove.

\section{Acknowledgments}

P.E. is grateful for the hospitality extended to him at the Kochi University of Technology where the idea of the approximation was formulated. The research was supported by the Czech Ministry of Education, Youth and Sports within the project LC06002 and by the Japanese Ministry of Education, Culture, Sports, Science and Technology under the Grant number 21540402. 


\section{References}

[1] K. Ruedenberg and C.W. Scherr, Free-electron network model for conjugated systems, I. Theory, J. Chem. Phys. 21 (1953), 1565-1581.

[2] P. Exner, J.P. Keating, P. Kuchment, T. Sunada, A. Teplyaev, eds.: Analysis on Graphs and Applications, Proceedings of a Isaac Newton Institute programme, January 8-June 29, 2007; 670 p.; AMS "Proceedings of Symposia in Pure Mathematics" Series, vol. 77, Providence, R.I., 2008

[3] T. Cheon and T. Shigehara: Realizing discontinuous wave functions with renormalized short-range potentials, Phys. Lett. A243 (1998), 111-116.

[4] P. Exner, H. Neidhardt and V.A. Zagrebnov: Potential approximations to $\delta^{\prime}$ : an inverse Klauder phenomenon with norm-resolvent convergence, Commun. Math. Phys. 224 (2001), 593-612.

[5] T. Cheon and P. Exner, An approximation to delta' couplings on graphs, J. Phys. A: Math. Gen. 37 (2004), L329335.

[6] P. Exner and O. Turek: Approximations of singular vertex couplings in quantum graphs, Rev. Math. Phys. 19 (2007), 571-606

[7] T. Shigehara, H. Mizoguchi, T. Mishima, T. Cheon: Realization of a four parameter family of generalized onedimensional contact interactions by three nearby delta potentials with renormalized strengths, IEICE Trans. Fund. Elec. Comm. Comp. Sci. E82-A (1999), 1708-1713.

[8] P. Exner, O. Post: Approximation of quantum graph vertex couplings by scaled Schrödinger operators on thin branched manifolds, arXiv: $0811.3707 \mathrm{v} 1$

[9] V. Kostrykin, R. Schrader: Kirchhoff's rule for quantum wires, J. Phys. A: Math. Gen. 32 (1999), 595-630.

[10] M. Harmer: Hermitian symplectic geometry and extension theory, J. Phys. A: Math. Gen. 33 (2000), 9193-9203

[11] V. Kostrykin, R. Schrader: Kirchhoff's rule for quantum wires. II: The Inverse Problem with Possible Applications to Quantum Computers, Fortschr. Phys. 48 (2000), 703-716.

[12] V.I. Gorbachuk, M.L. Gorbachuk: Boundary value problems for operator differential equations, Kluwer, Dordrecht 1991.

[13] T. Fülőp, I. Tsutsui: A free particle on a circle with point interaction, Phys. Lett. A264 (2000), 366-374

[14] P. Exner and P. Šeba: Free quantum motion on a branching graph, Rep. Math. Phys. 28 (1989), 7-26.

[15] P. Kuchment: Quantum graphs: I. Some basic structures, Waves Random Media 14 (2004), S107-S128.

[16] S. Albeverio, F. Gesztesy, R. Høegh-Krohn and H. Holden, Solvable Models in Quantum Mechanics, 2nd edition, AMS Chelsea, 2005. 